\title{
Multilevel particle filters
}

DOI:

10.1137/17M1111553

\section{Document Version}

Accepted author manuscript

Link to publication record in Manchester Research Explorer

\section{Citation for published version (APA):}

Jasra, A., Kamatani, K., Law, K. J. H., \& Zhou, Y. (2017). Multilevel particle filters. SIAM JOURNAL ON NUMERICAL ANAL YSIS, 55(6), 3068-3096. https://doi.org/10.1137/17M1111553

\section{Published in:}

SIAM JOURNAL ON NUMERICAL ANALYSIS

\section{Citing this paper}

Please note that where the full-text provided on Manchester Research Explorer is the Author Accepted Manuscript or Proof version this may differ from the final Published version. If citing, it is advised that you check and use the publisher's definitive version.

\section{General rights}

Copyright and moral rights for the publications made accessible in the Research Explorer are retained by the authors and/or other copyright owners and it is a condition of accessing publications that users recognise and abide by the legal requirements associated with these rights.

\section{Takedown policy}

If you believe that this document breaches copyright please refer to the University of Manchester's Takedown Procedures [http://man.ac.uk/04Y6Bo] or contact uml.scholarlycommunications@manchester.ac.uk providing relevant details, so we can investigate your claim.

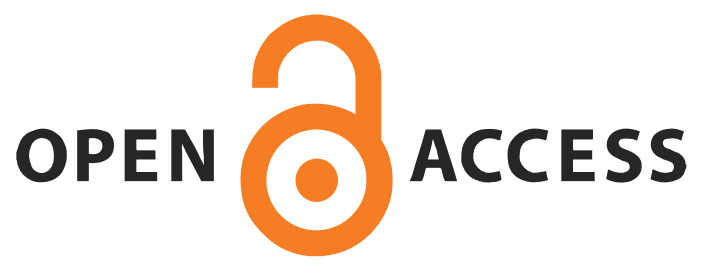




\title{
MULTILEVEL PARTICLE FILTERS
}

\author{
AJAY JASRA*, KENGO KAMATANI ${ }^{\dagger}$, KODY J. H. LAW ${ }^{\ddagger}$, AND YAN ZHOU
}

\begin{abstract}
In this paper the filtering of partially observed diffusions, with discrete-time observations, is considered. It is assumed that only biased approximations of the diffusion can be obtained, for choice of an accuracy parameter indexed by $l$. A multilevel estimator is proposed, consisting of a telescopic sum of increment estimators associated to the successive levels. The work associated to $\mathcal{O}\left(\varepsilon^{2}\right)$ mean-square error between the multilevel estimator and average with respect to the filtering distribution is shown to scale optimally, for example as $\mathcal{O}\left(\varepsilon^{-2}\right)$ for optimal rates of convergence of the underlying diffusion approximation. The method is illustrated on some toy examples as well as estimation of interest rate based on real S\&P 500 stock price data.
\end{abstract}

Key words: Filtering; Diffusions; Particle Filter; Multilevel Monte Carlo

1. Introduction. Problems which involve continuum fields are typically discretized before they are solved numerically. Finer resolution solutions are more expensive to compute than coarse resolution ones. Often such discretizations naturally give rise to resolution hierarchies, for example nested meshes. Successive solution on refined meshes can be utilized to mitigate the number of necessary solves at the finest resolution. For solution of linear systems, the coarsened systems are solved as pre-conditioners within the framework of iterative linear solvers in order to reduce the condition number, and hence the number of necessary iterations, at the fine resolution. This is the principle of multi-grid methods [4].

In the context of Monte Carlo methods, a telescoping sum of correlated differences at successive refinement levels can be utilized so that the bias of the resulting multilevel estimator is determined by the finest level but the variance is given by the sum of the variances of the increments. The decay in the variance of the increments of finer levels means that the number of samples required to reach a given error tolerance is also reduced for finer levels. This can then be optimized to balance the extra per-sample cost at the finer levels $[19,14,15]$.

This article is focused on the filtering problem. This means there is a pair of processes in time, one of which is a hidden Markov chain (in our case a diffusion process), and this is associated to the other process, which represents discretely observed data. Exact filtering is seldom possible and one often has to resort to numerical methods, such as Monte Carlo. It is well known in the literature that simple Monte Carlo strategies involving ratios of likelihood-weighted integrals tend to converge slowly and be inefficient, see for instance [12]. Perhaps the best known methodology to accurately approximate the filtering distribution is the particle filter (see e.g. [12]). This method generates a collection of weighted samples (or particles) in parallel, recursively in time. The weighted particles are propagated at each time by a sampling step and are then re-weighted in order to account for the new observation. It is well-known that this procedure suffers from the so-called weight degeneracy problem, where eventually one particle will have a weight very close to 1 and all the others will have negligible weight,

*Department of Statistics \& Applied Probability, National University of Singapore, Singapore, 117546, SG.

${ }^{\dagger}$ Graduate School of Engineering Science, Osaka University, Osaka, 565-0871, JP.

${ }^{\ddagger}$ Computer Science and Mathematics Division, Oak Ridge National Laboratory, Oak Ridge, 37934 TN, USA.

$\S$ Department of Statistics \& Applied Probability, National University of Singapore, Singapore, 117546, SG. 
meaning the effective sample size is 1 . Incorporating a resampling step mitigates this problem. The methodology is well understood, and several convergence results exist; see for instance [7]. In particular, it is well-known that under various conditions, the error (for instance measured in $\mathbb{L}_{p}$ ) will not depend upon the time parameter; see e.g. $[7,9]$. The required conditions are often associated to the ergodicity of the hidden chain and regularity properties of the weights, such as boundedness.

The natural and yet challenging extension of the multilevel Monte Carlo (MLMC) framework to inference problems has recently been pioneered by the works $[20,26,3$, $21,18]$. Rigorous results for consistent filtering using the MLMC framework within particle filtering have yet to be obtained. In this article, the context of a partially observed diffusion is considered, with observations in discrete time; this will be detailed explicitly in the next section. The works $[18,17]$ recently proposed to use the MLMC idea in particle filters by leveraging a deterministic resampling mechanism, and numerical results indicate that this can lead to the desired improvement in efficiency in some cases. However, there are no rigorous convergence results and the cost of that resampling step itself can be a problem.

In the context of filtering, one difficulty is the nonlinearity of the update, which precludes the construction of unbiased estimators, at least with the currently available methodology. However, this problem was already addressed in [3]. Indeed some ingenuity is required to successfully actualize the necessary resampling step while retaining adequate correlations. In this paper a novel coupled resampling procedure is analyzed, which enables this extension of the MLMC framework to the multilevel particle filter (MLPF). The work associated to $\mathcal{O}\left(\varepsilon^{2}\right)$ mean-square error between the multilevel estimator and average with respect to the filtering distribution is shown to scale optimally, for example as $\mathcal{O}\left(\varepsilon^{-2}\right)$ for optimal rate of convergence of the underlying diffusion approximation. We remark that our rigorous results depend upon the time parameter, whereas our numerical examples indicate time-uniform convergence of the error. To obtain rigorous time-uniform results, one must very precisely analyze the stability of the coupled resampling operator introduced here. This challenging task is left for future work to keep the length of this article reasonable.

This new MLPF algorithm is illustrated on some toy diffusion examples, as well as a stochastic volatility model with real S\&P 500 stock price data. The performance of the new algorithm easily reaches an order of magnitude or greater improvement in cost, and the theoretical rate is verified so that improvement will continue to amplify as more accurate estimates are obtained. The method is also broadly applicable to a wide range of problems in data assimilation [28], for example meteorology and subsurface geosciences. Furthermore, the method is very amenable to parallelization strategies, leaving open great potential for its use on next generation super-computers.

2. Set Up. We consider the following partially-observed diffusion process:

$$
d X_{t}=a\left(X_{t}\right) d t+b\left(X_{t}\right) d W_{t}
$$

with $X_{t} \in \mathbb{R}^{d}, t \geq 0, X_{0}$ having (possibly) degenerate distribution $\eta_{0}, a: \mathbb{R}^{d} \rightarrow \mathbb{R}^{d}$ (denote the $j^{t h}$-element as $a^{j}\left(X_{t}\right)$ ), $b: \mathbb{R}^{d} \rightarrow \mathbb{R}^{d \times d}$ (denote the $j^{\text {th }}, k^{t h}$-element as $\left.b^{j, k}\left(X_{t}\right)\right)$ and $\left\{W_{t}\right\}_{t \in[0, T]}$ a Brownian motion of $d$-dimensions. The following assumptions will be made on the diffusion process.

Assumption 2.1. The coefficients $a^{j}(x), b^{j, k}(x)$ are twice continuously differentiable with bounded derivatives $\left(x \in \mathbb{R}^{d}\right)$, for $j, k=1, \ldots, d$. Also, a and $b$ satisfy

(i) uniform ellipticity: $b(x) b(x)^{T}$ is uniformly positive definite; 
(ii) globally Lipschitz: there is a $C>0$ such that $\left|a^{j}(x)-a^{j}(y)\right|+\mid b^{j, k}(x)-$ $b^{j, k}(y)|\leq C| x-y \mid$ for all $x, y \in \mathbb{R}^{d}$ and $j, k \in\{1, \ldots, d\}$;

(iii) boundedness: $\mathbb{E}\left|X_{0}\right|^{p}<\infty$ for all $p \geq 1$.

Notice that (ii) and (iii) together imply that $\mathbb{E}\left|X_{t}\right|^{p}<\infty$ for all $t$, where $\left(X_{t}\right.$ is the solution of (2.1)).

It will be assumed that the data are regularly spaced (i.e. in discrete time) observations $y_{1}, \ldots, y_{n}$, where $y_{n} \in \mathbb{R}^{m}$ is a realization of $Y_{n}$ and $Y_{n} \mid X_{n \delta}$ has density given by $G\left(y_{n}, x_{n \delta}\right)$. For simplicity of notation let $\delta=1$ (which can always be done by rescaling time), so $X_{n}=X_{n \delta}$. The joint probability density of the observations and the unobserved diffusion at the observation times is then

$$
\prod_{i=1}^{n} G\left(y_{i}, x_{i}\right) Q^{\infty}\left(x_{(i-1)}, x_{i}\right)
$$

where $Q^{\infty}\left(x_{(i-1)}, x\right)$ is the transition density of the diffusion process as a function of $x$, i.e. the density of the solution $X_{1}$ of Eq. (2.1) at time 1 given initial condition $X_{0}=x_{(i-1)}$.

The following assumptions will be made on the observations.

Assumption 2.2 (Observation properties). There are some $c>1$ and $C>0$, such that $G$ satisfies

(i) boundedness: $c^{-1}<G(y, x)<c$ for all $x \in \mathbb{R}^{d}$ and $y \in \mathbb{R}^{m}$;

(ii) globally Lipschitz: for all $y \in \mathbb{R}^{m},\left|G(y, x)-G\left(y, x^{\prime}\right)\right| \leq C\left|x-x^{\prime}\right|$.

The objective is to approximate the filtering distribution $\pi^{\infty}\left(x_{n} \mid y_{1: n}\right)$, and the forecast distribution $\pi^{\infty}\left(x_{n} \mid y_{1: n-1}\right)$, which will be denoted $\widehat{\eta}_{n}^{\infty}$ and $\eta_{n}^{\infty}$, respectively. With a particle filter one obtains a collection of samples $\left\{u_{n}^{\infty, i}\right\}_{i=1}^{N}$ with associated weights $\left\{\omega_{n}^{\infty, i}\right\}_{i=1}^{N}$, giving rise to an empirical measure

$$
\widehat{\eta}_{n}^{\infty, N}=\sum_{i=1}^{N} \omega_{n}^{\infty, i} \delta_{u_{n}^{\infty, i}}
$$

which approximates $\widehat{\eta}_{n}^{\infty}$, and similar for $\eta_{n}^{\infty}$. The particle filter works by interlacing importance sampling for the Bayesian updates incorporating observations, with a resampling selection step to rejuvenate the ensemble, and a mutation move which propagates the ensemble forward through the diffusion (e.g. [12] and the references therein). It is a well-known fact that if $Q^{\infty}(x, \cdot)$ can be sampled from exactly, then the particle filter achieves standard convergence rates for Monte Carlo approximation of expectations of quantities of interest $\varphi \in \mathcal{B}_{b}\left(\mathbb{R}^{d}\right)$, the set of bounded measurable functions over $\mathbb{R}^{d}[5]$ :

$$
\mathbb{E}\left|\widehat{\eta}_{n}^{\infty, N}(\varphi)-\widehat{\eta}_{n}^{\infty}(\varphi)\right|^{2} \leq C / N
$$

where the shorthand notation $\mu(\varphi)=\int_{\mathbb{R}^{d}} \varphi(u) \mu(d u)$ is used for any probability $\mu$ over $\mathbb{R}^{d}$. Note that $C$ often behaves poorly with respect to $d[1,2]$, but $C$ may not depend upon $n$; see [7]. In the setting considered in this paper:

1. It is (generally) not possible to sample exactly from $Q^{\infty}(x, \cdot)$.

2. The transition density is unavailable up-to a non-negative unbiased estimate. If either of these are possible, then alternative methods (e.g. [13]) may be more efficient.

It will be assumed that the diffusion process is approximated by a time-stepping method for time-step $h_{l}=2^{-l}$, and the corresponding forecast and filtering distributions will be denoted by $\eta_{n}^{l}$ and $\widehat{\eta}_{n}^{l}$. For simplicity and illustration, Euler's method 
[27] will be considered. However, the results can easily be extended and the theory will be presented more generally. In particular,

$$
\begin{aligned}
& X_{n,(k+1)}^{l}=X_{n, k}^{l}+h_{l} a\left(X_{n, k}^{l}\right)+\sqrt{h_{l}} b\left(X_{n, k}^{l}\right) \xi_{n, k}, \\
& \xi_{n, k} \stackrel{\text { i.i.d. }}{\sim} \mathcal{N}_{d}\left(0, I_{d}\right)
\end{aligned}
$$

for $k=0, \ldots, 2^{l}$, where $\mathcal{N}_{d}\left(0, I_{d}\right)$ is the $d$-dimensional normal distribution with mean zero and identity covariance (when $d=1$ the subscript is omitted). Note that $X_{n, m}^{l}$ corresponds to $X_{n+m 2^{-l}}^{l}$, where $X_{n}^{l}$ is the solution to the discretized diffusion at time $n$. The numerical scheme gives rise to its own transition density between observation times $Q^{l}\left(x_{(n-1)}, x\right)$, which is the density of $X_{(n-1), 2^{l}}^{l}=X_{n, 0}^{l}=X_{n}^{l}$, given initial condition $X_{(n-1), 0}^{l}=x_{(n-1)}$. Let $\eta_{1}^{l}(\varphi):=\mathbb{E} \varphi\left(X_{1}^{l}\right)$ for $l=0, \ldots, \infty$. Suppose one aims to approximate the expectation of $\varphi \in \mathcal{B}_{b}\left(\mathbb{R}^{d}\right)$. For a given $L$, the Monte Carlo approximation of $\eta_{1}^{\infty}(\varphi)$ by

$$
\eta_{1}^{L, N}(\varphi)=\frac{1}{N} \sum_{i=1}^{N} \varphi\left(X_{1}^{L, i}\right), \quad X_{1}^{L, i} \sim Q^{L}\left(x_{0}, \cdot\right),
$$

has mean square error (MSE) given by

$$
\mathbb{E}\left|\eta_{1}^{L, N}(\varphi)-\eta_{1}^{\infty}(\varphi)\right|^{2}=\underbrace{\mathbb{E}\left|\eta_{1}^{L, N}(\varphi)-\eta_{1}^{L}(\varphi)\right|^{2}}_{\text {variance }}+\underbrace{\left|\eta_{1}^{L}(\varphi)-\eta_{1}^{\infty}(\varphi)\right|^{2}}_{\text {bias }} .
$$

If one aims for $\mathcal{O}\left(\varepsilon^{2}\right) \mathrm{MSE}$ with optimal cost, then one must balance these two terms.

For $l=0,1, \ldots, L$, the hierarchy of time-steps $\left\{h_{l}\right\}_{l=0}^{L}$ gives rise to a hierarchy of transition densities $\left\{Q^{l}\right\}_{l=0}^{L}$. In this context, for a single transition, it is wellknown that the multilevel Monte Carlo (MLMC) method $[14,19]$ can reduce the cost to obtain a given level of mean-square error (MSE) (2.4). The description of this method and its extension to the particle filter setting will be the topic of the next section.

3. Multilevel Particle Filters. In this section, the multilevel particle filter will be introduced. First, a review of the standard multilevel Monte-Carlo method is presented, illustrating the strategy for reducing the necessary cost for a given level of mean-square error. Next, the extension to the multilevel particle filter is presented.

3.1. Multilevel Monte Carlo. The standard multilevel Monte Carlo (MLMC) framework [14] begins with asymptotic estimates for weak and strong error rates, and the associated cost. In particular, assume the following.

Assumption 3.1 (MLMC Rates). There are $\alpha, \beta, \gamma>0$ such that

(i) $\left|\mathbb{E}\left[\varphi\left(X_{1}^{l}\right)-\varphi\left(X_{1}^{\infty}\right)\right]\right|=\mathcal{O}\left(h_{l}^{\alpha}\right)$;

(ii) For $p \geq 1, \mathbb{E}\left[\left|\varphi\left(X_{1}^{l}\right)-\varphi\left(X_{1}^{\infty}\right)\right|^{p}\right]^{2 / p}=\mathcal{O}\left(h_{l}^{\beta}\right)$;

(iii) $\operatorname{COST}\left(X_{1}^{l}\right)=\mathcal{O}\left(h_{l}^{-\gamma}\right)$,

where COST denotes the computational effort to obtain one sample $X_{1}^{l}$, and $h_{l}$ is the grid-size of the numerical method, for example the Euler method as given in (2.3). In this case $\alpha=\beta=\gamma=1$. In general $\alpha \geq \beta / 2$, as the choice $\alpha=\beta / 2$ is always possible, by Jensen's inequality.

Recall that in order to minimize the effort to obtain a given MSE, one must balance the terms in (2.4). Based on Assumption 3.1(i) above, a bias error proportional to $\varepsilon$ will require

$$
L(\varepsilon) \propto-\log (\varepsilon) /(\log (2) \alpha) .
$$


The associated cost, in terms of $\varepsilon$, for a given sample is $\mathcal{O}\left(\varepsilon^{-\gamma / \alpha}\right)$. Furthermore, the necessary number of samples to obtain a variance proportional to $\varepsilon^{2}$ for this standard single level estimator is given by $N \propto \varepsilon^{-2}$ following from (2.2). So the total cost to obtain a mean-square error tolerance of $\mathcal{O}\left(\varepsilon^{2}\right)$ is: \#samples $\times($ cost/sample $)=$ total $\operatorname{cost} \propto \varepsilon^{-2-\gamma / \alpha}$. To anchor to the particular example of the Euler-Maruyama method, the total cost is $\mathcal{O}\left(\varepsilon^{-3}\right)$.

Define a kernel $M^{l}:\left[\mathbb{R}^{d} \times \mathbb{R}^{d}\right] \times\left[\sigma\left(\mathbb{R}^{d}\right) \times \sigma\left(\mathbb{R}^{d}\right)\right] \rightarrow \mathbb{R}_{+}$, where $\sigma(\cdot)$ denotes the sigma algebra of measurable subsets, such that $M_{1}^{l}(x, A):=M^{l}\left(\left[x, x^{\prime}\right], A \times \mathbb{R}^{d}\right)=$ $Q^{l}(x, A)$ and $M_{2}^{l}\left(x^{\prime}, A\right):=M^{l}\left(\left[x, x^{\prime}\right], \mathbb{R}^{d} \times A\right)=Q^{l-1}\left(x^{\prime}, A\right)$. The idea of MLMC is the following. First approximate the $l^{\text {th }}$ increment $\left(\eta_{1}^{l}-\eta_{1}^{l-1}\right)(\varphi)$ by an empirical average

$$
Y_{l}^{N_{l}}(\varphi):=\frac{1}{N_{l}} \sum_{i=1}^{N_{l}} \varphi\left(X_{1,1}^{l, i}\right)-\varphi\left(X_{1,2}^{l, i}\right)
$$

where $\left[X_{1,1}^{l, i}, X_{1,2}^{l, i}\right] \sim M^{l}\left(\left[x_{0}, x_{0}\right], \cdot\right)$, given initial datum $X_{0}=x_{0}$. The multilevel estimator is a telescopic sum of such unbiased increment estimators, which yields an unbiased estimator of $\eta_{1}^{L}(\varphi)$. It can be defined in terms of its empirical measure as

$$
\eta_{1}^{L, \operatorname{Multi}}(\varphi):=\sum_{l=0}^{L} Y_{l}^{N_{l}}(\varphi),
$$

under the convention that $\varphi\left(X_{1,2}^{0, i}\right) \equiv 0$.

The mean-square error of the multilevel estimator is given by

$$
\begin{aligned}
& \mathbb{E}\left\{\eta_{1}^{L, \text { Multi }}(\varphi)-\eta_{1}^{\infty}(\varphi)\right\}^{2}= \\
& \underbrace{\sum_{l=0}^{L} \mathbb{E}\left\{Y_{l}^{N_{l}}(\varphi)-\left[\eta_{1}^{l}(\varphi)-\eta_{1}^{l-1}(\varphi)\right]\right\}^{2}}_{\text {variance }}+\{\underbrace{\eta_{1}^{L}(\varphi)-\eta_{1}^{\infty}(\varphi)}_{\text {bias }}\}^{2} .
\end{aligned}
$$

The key observation is that the bias is given by the finest level, whilst the variance is decomposed into a sum of variances of the increments $\mathcal{V}=\sum_{l=0}^{L} V_{l} N_{l}^{-1}$. Sufficient correlation must be built into the kernels $M^{l}$ to ensure condition Assumption 3.1(ii)above carries over to the increments (for example two discretizations of the same random realization of the SDE (2.1)). Then the variance of the $l^{\text {th }}$ increment has the form $V_{l} N_{l}^{-1}$ and $V_{l}=\mathcal{O}\left(h_{l}^{\beta}\right)$ following from Assumption 3.1 (ii), allowing smaller number of samples $N_{l}$ at cost $C_{l}=\mathcal{O}\left(h_{l}^{-\gamma}\right)$ for larger $l$, following from Assumption 3.1(iii). The total cost is given by the $\operatorname{sum} \mathcal{C}=\sum_{l=0}^{L} C_{l} N_{l}$. Based on Assumption 3.1(ii) and Assumption 3.1(iii) above, optimizing $\mathcal{C}$ for a fixed $\mathcal{V}$ yields that $N_{l}=\lambda^{-1 / 2} 2^{-(\beta+\gamma) l / 2}$, for Lagrange multiplier $\lambda$. In the Euler-Maruyama case $N_{l}=\lambda^{-1 / 2} 2^{-l}$. Now, one can see that after fixing the bias to $c \varepsilon$, one aims to find the Lagrange multiplier $\lambda$ such that $\mathcal{V} \approx c^{2} \varepsilon^{2}$. Defining $N_{0}=\lambda^{-1 / 2}$, then $\mathcal{V}=N_{0}^{-1} \sum_{l=0}^{L} 2^{(\gamma-\beta) l / 2}$, so one must have $N_{0} \propto \varepsilon^{-2} K(\varepsilon)$, where $K(\varepsilon)=\sum_{l=0}^{L} \sqrt{V_{l} C_{l}}=\sum_{l=0}^{L} 2^{(\gamma-\beta) l / 2}$, and the $\varepsilon$-dependence comes from $L(\varepsilon)$, as defined in (3.1). There are three cases, with associated $K$, and hence cost $\mathcal{C}$, given in Table 3.1 .

For example, Euler-Maruyama falls into the case $(\beta=\gamma)$, so that $\mathcal{C}(\varepsilon)=\mathcal{O}\left(\varepsilon^{-2} \log (\varepsilon)^{2}\right)$. In this case, one chooses $N_{0}=C \varepsilon^{-2}|\log (\varepsilon)|=C 2^{2 L} L$, where the purpose of $C$ is to match the variance with the $\operatorname{bias}^{2}$, similar to the single level case. 


\begin{tabular}{|c||c|c|}
\hline CASE & $K(\varepsilon)$ & $\mathcal{C}(\varepsilon)$ \\
\hline \hline$\beta>\gamma$ & $\mathcal{O}(1)$ & $\mathcal{O}\left(\varepsilon^{-2}\right)$ \\
\hline$\beta=\gamma$ & $\mathcal{O}(-\log (\varepsilon))$ & $\mathcal{O}\left(\varepsilon^{-2} \log (\varepsilon)^{2}\right)$ \\
\hline$\beta<\gamma$ & $\mathcal{O}\left(\varepsilon^{(\beta-\gamma) /(2 \alpha)}\right)$ & $\mathcal{O}\left(\varepsilon^{-2+(\beta-\gamma) / \alpha}\right)$ \\
\hline
\end{tabular}

Table 3.1: The three cases of multilevel Monte Carlo, and associated constant $K(\varepsilon)$ and $\operatorname{cost} \mathcal{C}(\varepsilon)$.

The kernel $M^{l}$ can be constructed using the following strategy. First the finer discretization is simulated using (2.3) (ignoring index $n$ ) with $X_{0,1}^{l, i}=x_{0}$, for $i \in$ $\left\{1, \ldots, N_{l}\right\}$. Now for the coarse discretization, let $X_{0,2}^{l, i}=x_{0}$ for $i \in\left\{1, \ldots, N_{l}\right\}$, let $h_{l-1}=2 h_{l}$ and for $k \in\left\{1, \ldots, 2^{l-1}\right\}$ simulate

$$
X_{k+1,2}^{l, i}=X_{k, 2}^{l, i}+h_{l-1} a\left(X_{k, 2}^{l, i}\right)+\sqrt{h_{l-1}} b\left(X_{k, 2}^{l, i}\right)\left(\xi_{2 k}^{i}+\xi_{2 k+1}^{i}\right),
$$

where $\left\{\xi_{k}^{i}\right\}_{i=1, k=0}^{N_{l}, 2^{l}}$ are the $i^{\text {th }}$ realizations used in the simulation of the finer discretization. This procedure defines a kernel $M^{l}$ as above, such that $\left(X_{2^{l-1}, 1}^{l, i}, X_{2^{l-1,2}}^{l, i}\right) \sim$ $M^{l}\left(\left[x_{0}, x_{0}\right], \cdot\right)$ are suitably coupled and the standard MLMC theory will go through with $\alpha=\beta=\gamma=1$ above.

3.2. Multilevel Particle Filters. The framework of the previous section will now be extended to the new multilevel particle filter (MLPF). Throughout, the observations $y_{1: n}, n \geq 1$ are omitted from the notations. It will be convenient to define $U_{n}^{l}:=X_{n}^{l} \mid y_{1: n-1}$ for $l=0, \ldots, \infty$, with $U_{n}^{\infty}:=X_{n}^{\infty} \mid y_{1: n-1}$ denoting the limiting continuous-time process, and denote the associated predictive distributions by $\eta_{n}^{l}$. It will also be useful to define $\widehat{U}_{n}^{l}:=X_{n}^{l} \mid y_{1: n}$, and its distribution $\widehat{\eta}_{n}^{l}$. Let $\varphi \in \mathcal{B}_{b}\left(\mathbb{R}^{d}\right)$ and consider the following decomposition

$$
\widehat{\eta}_{n}^{\infty}(\varphi)=\sum_{l=0}^{L}\left(\widehat{\eta}_{n}^{l}-\widehat{\eta}_{n}^{l-1}\right)(\varphi)+\left(\widehat{\eta}_{n}^{\infty}-\widehat{\eta}_{n}^{L}\right)(\varphi)
$$

where $\eta_{n}^{-1}(\varphi):=0$.

Let $U_{0,1}^{l, i}=\widehat{U}_{0,1}^{l, i}=U_{0,2}^{l, i}=\widehat{U}_{0,2}^{l, i}=X_{0}^{i}$, where $X_{0}^{i} \sim \eta_{0}=\widehat{\eta}_{0}$, and iterate the following. Draw $\left[U_{n, 1}^{l, i}, U_{n, 2}^{l, i}\right] \sim M^{l}\left(\left[\widehat{U}_{n-1,1}^{l, i}, \widehat{U}_{n-1,2}^{l, i}\right]\right.$, . $)$ Each summand in the first term of (3.6) can be estimated with:

$$
\sum_{i=1}^{N_{l}}\left\{w_{n, 1}^{l, i} \varphi\left(U_{n, 1}^{l, i}\right)-w_{n, 2}^{l, i} \varphi\left(U_{n, 2}^{l, i}\right)\right\}
$$

where the weights are defined as follows, for $j \in\{1,2\}$,

$$
w_{n, j}^{l, i}=\frac{G\left(y_{n}, U_{n, j}^{l, i}\right)}{\sum_{h=1}^{N_{l}} G\left(y_{n}, U_{n, j}^{l, h}\right)} .
$$

It is clear that for suitably well-behaved $G$, for example satisfying Assumption 2.2, such an estimate will satisfy the standard MLMC identity and cost. However, it is wellknown that one must perform resampling in order for a particle filter to perform well 


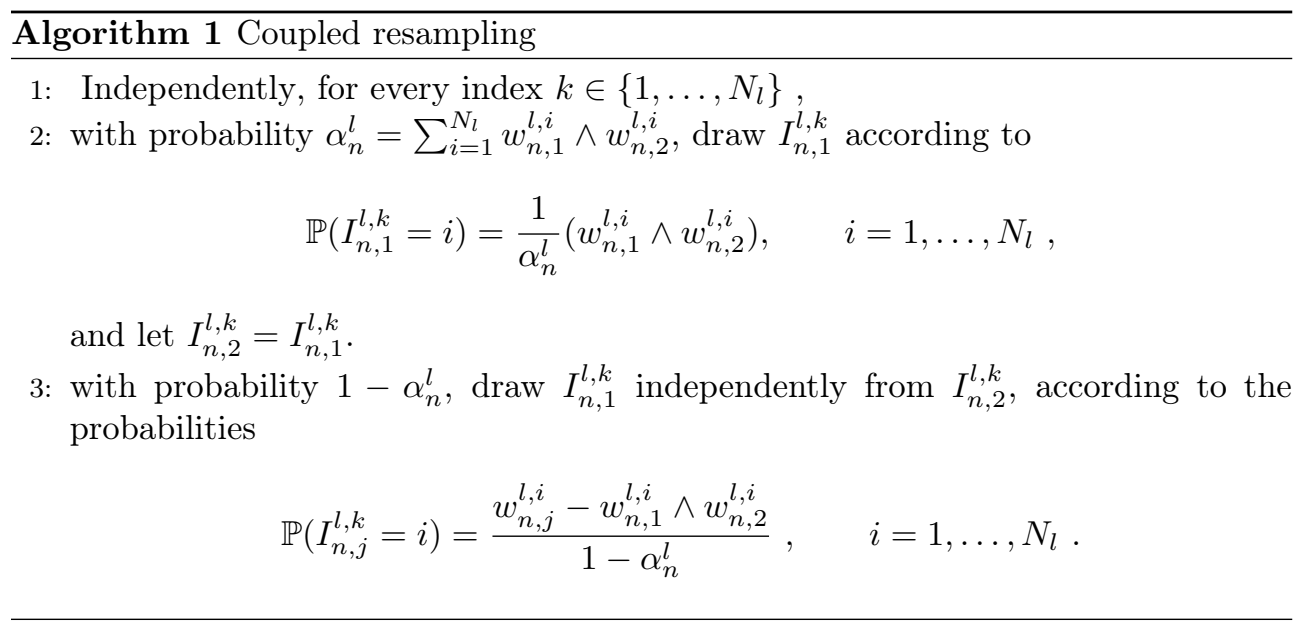

for multiple steps. Here this is a particularly challenging point, as the samples have to remain suitably coupled after the resampling, so that similar rates hold as above. The indices $I_{n, j}^{l, k}, j \in\{1,2\}$, are sampled according to the coupled resampling procedure described (see also [6]) in Algorithm 1.

The indices for the fine (resp. coarse) discretization are resampled marginally according to $w_{n, 1}^{l, i}$ (resp. $w_{n, 2}^{l, i}$ ), which is exactly as required. Note that this is the coupling which maximizes the probability $\alpha_{n}^{l}$ that the indices are the same. Therefore, pairwise correlations due to the standard coupled evolution step will be preserved perfectly with the maximum probability. Notice that it is necessary to independently sample the fine and coarse levels with a small probability in order to preserve the marginals. However, it will be shown that the resulting samples do remain sufficiently coupled, although with a slightly lower rate than the vanilla MLMC. As mentioned in Sections 1 and 6, there are some alternative methods to coupled resampling, for instance as in $[18,17]$. The methods in $[18,17]$ do resampling using a deterministic transformation linked to optimal transportation (see also [30]). This resampling transformation itself can be prohibitively expensive, but in examples where this is not the case it has been numerically shown to preserve the correct rate.

Finally the multilevel particle filter (MLPF) is given in Algorithm 2. To ease the notation, the case $l=0$ should be understood as the ordinary particle filter which targets $\widehat{\eta}_{n}^{0}$.

The method is completed by (i) identifying $L$ and $\left\{N_{l}\right\}_{l=0}^{L}$ for use in Algorithm 2, and (ii) constructing the multilevel estimator as in Equation (4.2) below using the multilevel ensemble output from Algorithm 2. We iterate that for the MLMC identity to pay off optimally, it suffices to choose $L=|\log \varepsilon| / \alpha \log 2$, where $\alpha$ is the weak rate of convergence, and

$$
N_{l}=\varepsilon^{-2} \sqrt{V_{l} / C_{l}} K(\varepsilon),
$$

where we recall that in general $K(\varepsilon)=\sum_{l=0}^{L} \sqrt{V_{l} C_{l}}$. Typically one can estimate the $\operatorname{cost} C_{l}$ without simulation, but simulation can be done to verify the cost scaling of a single simulation at each level or just a pair of levels. If one has a priori estimates for the rate of convergence of $V_{l}$ and the bias rate $\alpha$, then these can be used. Otherwise, these rates (and the associated constants) can be estimated using a pair (or more) of levels as well, and one or a few coupled resampling steps. Note that the coupled 


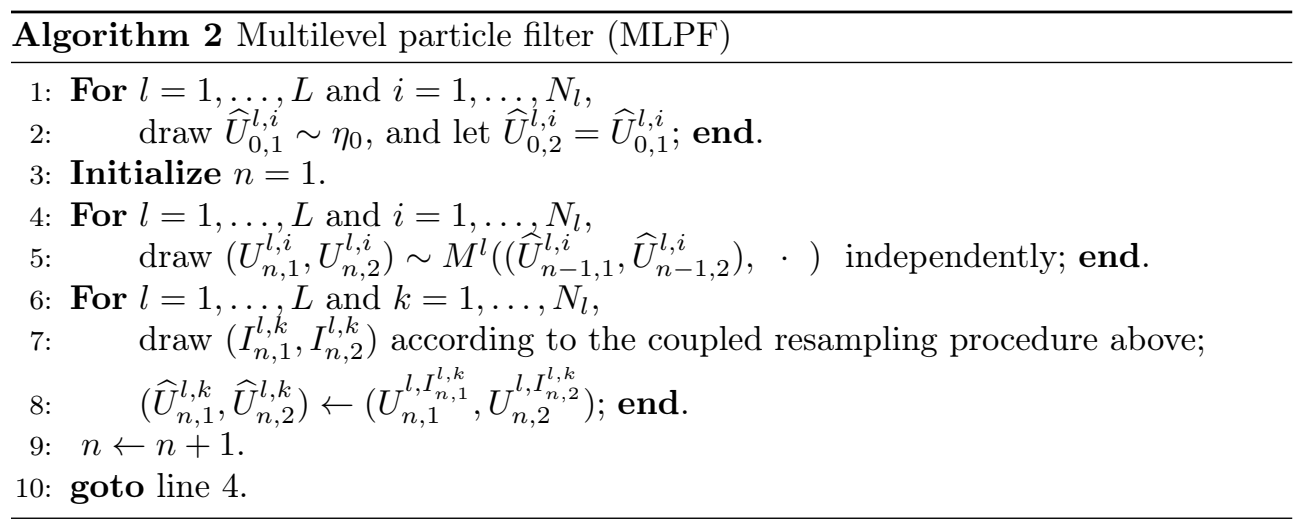

resampling must be done, as the rate is affected by it. Indeed for Euler's method as considered here, and $\beta$ defined in Assumption 4.2, $V_{l}=2^{l \beta / 2}$ (rather than $2^{l \beta}$ for the evolution alone).

Note that if the variance of the weights becomes substantial, one can use the approach in [22] to deal with this issue.

4. Theoretical Results. The calculations leading to the results in this section are performed via a Feynman-Kac type representation (see [7, 8]) which is detailed in the supplementary material. Denote the marginal transition kernels of the Euler discretization procedure described above at level $l$ as $M_{1}^{l}$ (fine) and $M_{2}^{l}$ (coarse). Note that these results do not depend on Euler discretization and hold for any general coupled particle filter. Also note that the results are easily extended to non-autonomous SDE (2.1) (that is, the drift and diffusion coefficients are functions of time), at the expense of additional technicalities. We remark that our analysis only applies to the case where one resamples at each time. However, such analysis can be extended to the case where one resamples according to the effective sample size (which is used in the numerical simulations), i.e. at stochastic times, for example using the ideas in [10]. For simplicity, the deterministic resampling approach is employed, since it simplifies what are already long and detailed calculations. We expect the results to hold for stochastic resampling strategies as well, which can result in lower variance estimators and can therefore be preferable in practice. The predictor at time $n$, level $l$, is denoted as $\eta_{n, 1}^{l}$ (fine) and $\eta_{n, 2}^{l}$ (coarse). $\mathcal{B}_{b}\left(\mathbb{R}^{d}\right)$ are the bounded, measurable and real-valued functions on $\mathbb{R}^{d}$ and $\operatorname{Lip}\left(\mathbb{R}^{d}\right)$ are the globally Lipschitz real-valued functions on $\mathbb{R}^{d}$. Denote the supremum norm as $\|\cdot\|$, and the total variation norm as $\|\cdot\|_{\text {tv }}$. For two Markov kernels $M_{1}$ and $M_{2}$ on the same space $E$, letting $\mathcal{A}=\{\varphi:\|\varphi\| \leq 1, \varphi \in \operatorname{Lip}(E)\}$ write

$$
||\left|M_{1}-M_{2}\right|||:=\sup _{\varphi \in \mathcal{A}} \sup _{x}\left|\int_{E} \varphi(y) M_{1}(x, d y)-\int_{E} \varphi(y) M_{2}(x, d y)\right| .
$$

Let $w_{n, j}^{l, i}$ denote the weights defined as in (3.7) with the index $n$ indicated explicitly. For each $j \in\{1,2\}, p \geq 1, m \geq 1$ define

$$
\begin{aligned}
& M_{m, j}\left(u_{p}, d u_{p+m}\right)= \\
& \int_{\mathbb{R}^{d \times m-1}} M_{j}\left(u_{p}, d u_{p+1}\right) \cdots M_{j}\left(u_{p+m-1}, d u_{p+m}\right) .
\end{aligned}
$$

Finally, the following notation is introduced for the selection densities $G_{n}(\cdot):=$ $G\left(y_{n+1}, \cdot\right)$. 
The following assumption will be made, uniformly over the level $l \in[0,1, \ldots)$, which will be omitted for notational simplicity.

Assumption 4.1 (Mutation). There exists a $C>0$ such that for each $u, u^{\prime} \in \mathbb{R}^{d}$, $j \in\{1,2\}$ and $\varphi \in \mathcal{B}_{b}\left(\mathbb{R}^{d}\right) \cap \operatorname{Lip}\left(\mathbb{R}^{d}\right)$

$$
\left|M_{j}(\varphi)(u)-M_{j}(\varphi)\left(u^{\prime}\right)\right| \leq C\|\varphi\|\left|u-u^{\prime}\right| .
$$

Additionally, it will be assumed that for all suitable test-functions $\varphi \in \mathcal{B}_{b}(\mathcal{U}) \cap$ $\operatorname{Lip}(\mathcal{U})$ the following hold.

Assumption 4.2 (MLPF rates). For $l \in[0,1, \ldots)$, and $p \geq 1$, let $\left(U_{1}^{l}, U_{2}^{l}\right) \sim$ $M^{l}\left(\left(U_{0,1}^{l}, U_{0,2}^{l}\right), \cdot\right)$, where $\left|\mathbb{E}\left[\varphi\left(U_{0,1}^{l}\right)-\varphi\left(U_{0,2}^{l}\right)\right]\right|=\mathcal{O}\left(h_{l}^{\alpha}\right)$ and $\mathbb{E}\left[\left|\varphi\left(U_{0,1}^{l}\right)-\varphi\left(U_{0,2}^{l}\right)\right|^{p}\right]^{2 / p}=$ $\mathcal{O}\left(h_{l}^{\beta}\right)$ for some $\alpha \geq \beta / 2>0$. Then, there is a $\gamma>0$ such that

(i) $\max \left\{\left|\mathbb{E}\left[\varphi\left(U_{1}^{l}\right)-\varphi\left(U_{2}^{l}\right)\right]\right|,||\left|M_{1}^{l}-M_{2}^{l}\right|||\right\}=\mathcal{O}\left(h_{l}^{\alpha}\right)$;

(ii) $\mathbb{E}\left[\left|\varphi\left(U_{1}^{l}\right)-\varphi\left(U_{2}^{l}\right)\right|^{p}\right]^{2 / p}=\mathcal{O}\left(h_{l}^{\beta}\right)$;

(iii) $\operatorname{COST}\left[M^{l}\right]=\mathcal{O}\left(h_{l}^{-\gamma}\right)$,

where $\operatorname{COST}\left[M^{l}\right]$ is the cost to simulate one sample from the kernel $M^{l}$.

4.1. Main Result. Here the MLPF theorem is presented, followed by the main theorem upon which it is based. The proof and supporting lemmas are provided in the supplementary materials. Let

$$
A_{l, n}^{N_{l}}(\varphi)=\sum_{i=1}^{N_{l}}\left[w_{n, 1}^{l, i} \varphi\left(U_{n, 1}^{l, i}\right)-w_{n, 2}^{l, i} \varphi\left(U_{n, 2}^{l, i}\right)\right],
$$

with the convention that $w_{n, 2}^{0, i}:=0$, and define

$$
\widehat{\eta}_{n}^{\mathrm{ML}}(\cdot):=\sum_{l=0}^{L} A_{l, n}^{N_{l}}(\cdot)
$$

Theorem 4.1 (MLPF). Let Assumptions 2.2, 4.1, and 4.2 be given and let $\gamma / \alpha \leq$ 2. Then for any $n \geq 0, \varphi \in \mathcal{B}_{b}\left(\mathbb{R}^{d}\right) \cap \operatorname{Lip}\left(\mathbb{R}^{d}\right)$, and $\varepsilon>0$, there exists a finite constant $C(n, \varphi)$, an $L>0$, and $\left\{N_{l}\right\}_{l=0}^{L}$ such that

$$
\mathbb{E}\left[\left(\widehat{\eta}_{n}^{\mathrm{ML}}(\varphi)-\widehat{\eta}_{n}^{\infty}(\varphi)\right)^{2}\right] \leq C(n, \varphi) \varepsilon^{2},
$$

for the cost $\mathcal{C}(\varepsilon)$ given in the third column of Table 4.1.

\begin{tabular}{|c||c|c|}
\hline CASE & $K(\varepsilon)$ & $\mathcal{C}(\varepsilon)$ \\
\hline \hline$\beta>2 \gamma$ & $\mathcal{O}(1)$ & $\mathcal{O}\left(\varepsilon^{-2}\right)$ \\
\hline$\beta=2 \gamma$ & $\mathcal{O}(-\log (\varepsilon))$ & $\mathcal{O}\left(\varepsilon^{-2} \log (\varepsilon)^{2}\right)$ \\
\hline$\beta<2 \gamma$ & $\mathcal{O}\left(\varepsilon^{(\beta-2 \gamma) /(4 \alpha)}\right)$ & $\mathcal{O}\left(\varepsilon^{-2+(\beta-2 \gamma) /(2 \alpha)}\right)$ \\
\hline
\end{tabular}

Table 4.1: The three cases of MLPF, and associated constant $K(\varepsilon)$ and $\operatorname{cost} \mathcal{C}(\varepsilon)$.

Proof. Notice that

$$
\begin{aligned}
\mathbb{E}\left[\left(\widehat{\eta}_{n}^{\mathrm{ML}}(\varphi)-\widehat{\eta}_{n}^{\infty}(\varphi)\right)^{2}\right] & \leq 2 \mathbb{E}\left[\left(\widehat{\eta}_{n}^{\mathrm{ML}}(\varphi)-\widehat{\eta}_{n}^{L}(\varphi)\right)^{2}\right] \\
& +2\left(\widehat{\eta}_{n}^{L}(\varphi)-\widehat{\eta}_{n}^{\infty}(\varphi)\right)^{2} .
\end{aligned}
$$


First, note that a theoretical kernel $M^{L, \infty}$ can be defined to generate coupled pairs of particles $\left(U_{n, 1}^{L, \infty}, U_{n, 2}^{L, \infty}\right)$ for $n \geq 1$ with marginals $U_{n, 1}^{L, \infty} \sim \widehat{\eta}_{n}^{\infty}$ and $U_{n, 2}^{L, \infty} \sim \widehat{\eta}_{n}^{L}$ satisfying the Assumptions 4.2. Assumption 2.2(i) then ensures the rate carries over to the update and finally induction shows the second term is $\mathcal{O}\left(h_{l}^{2 \alpha}\right)$. The rest of the proof follows from Theorems 4.2 and D.1, and Corollary D.1, noting that the terms in Corollary D. 1 are analogous to the $V_{l}$ terms from the standard multilevel theory described in the previous section. Therefore, upon choosing $L \propto-\log (\varepsilon)$, and $N_{l} \propto N_{0} 2^{-(\beta+2 \gamma) l / 4}$ with $N_{0} \propto \varepsilon^{-2} K(\varepsilon)$ and $K(\varepsilon)$ as in the second column of Table 4.1 , the results follow exactly as for MLMC above. Recall $K(\varepsilon)=\sum_{l=0}^{L} \sqrt{V_{l} C_{l}}$, which in this case is $\sum_{l=0}^{L} 2^{(2 \gamma-\beta) l / 4}$.

This Theorem can be immediately applied to the particular example of the diffusion (2.1), with appropriate discretization method. This is made explicit and precise in the following Corollary.

Corollary 4.1. Theorem 4.1 holds for the diffusion example (2.1) under Assumptions 2.1, given a numerical method which satisfies Assumptions 4.2. Furthermore Assumptions 4.2 hold for Euler-Maruyama method, with $\alpha=\beta=\gamma=1$. For a constant diffusion $b(x)=b$, one has $\beta=2$.

Proof. Assumptions 2.1 on (2.1) guarantee the required Assumptions 4.1 on the kernels $M^{L, \infty}$ [29]. For Euler-Maruyama method the kernels $M^{l}$ also satisfy Assumptions 4.1 and $4.2[16,11]$, and the rates can be found in [16, 27]. The improved rate $\beta=2$ for $b(x)=b$ is well-known, as the Euler method coincides with the Milstein method in the case of constant diffusion [16].

The main theorem which provides the appropriate convergence rate for the MLPF Theorem 4.1 is now presented.

TheOREM 4.2. Assume 4.1 for each level for the mutation kernel(s) and 2.2 for the updates. Then for any $n \geq 0,1 \leq L<+\infty, \varphi \in \mathcal{B}_{b}\left(\mathbb{R}^{d}\right) \cap \operatorname{Lip}\left(\mathbb{R}^{d}\right)$, there exists a constant $C(n, \varphi)=\max _{0 \leq l \leq L} C_{l}(n, \varphi)$ such that

$$
\begin{aligned}
\mathbb{E}\left[\left(\widehat{\eta}_{n}^{\mathrm{ML}}-\widehat{\eta}_{n}^{L}(\varphi)\right)^{2}\right] \leq \\
C(n, \varphi) \sum_{l=0}^{L} \frac{1}{N_{l}}\left(B_{l}(n)+\sum_{q \neq l=0}^{L} \frac{\sqrt{B_{l}(n) B_{q}(n)}}{N_{q}}\right) \\
B_{l}(n)=\left(\sum_{p=0}^{n} \mathbb{E}\left[\left\{\left|U_{p, 1}^{l, 1}-U_{p, 2}^{l, 1}\right| \wedge 1\right\}^{2}\right]^{1 / 2}+\left\|\eta_{p, 1}^{l}-\eta_{p, 2}^{l}\right\|_{t v}\right. \\
\left.\quad+\sum_{p=1}^{n}||\left|M_{p, 1}^{l}-M_{p, 2}^{l}\right|||\right)^{2} .
\end{aligned}
$$

Subscripts are added to indicate level-dependence, and the constants have been absorbed into the single one.

Proof. Let $\tilde{A}_{l, n}^{N_{l}}(\cdot)=\left(A_{l, n}^{N_{l}}-\left(\widehat{\eta}_{n}^{l}-\widehat{\eta}_{n}^{l-1}\right)\right)(\cdot)$, where $A_{l, n}^{N_{l}}$ is defined in Equation (4.1), with $\widehat{\eta}_{n}^{-1}:=0$. Noting the independence between increments, the telescoping 
sum provides

$$
\begin{aligned}
\mathbb{E}\left[\left(\sum_{l=0}^{L} \tilde{A}_{l, n}^{N_{l}}(\varphi)\right)^{2}\right] & =\sum_{l=0}^{L}\left(\mathbb{E}\left[\left(\tilde{A}_{l, n}^{N_{l}}(\varphi)\right)^{2}\right]\right. \\
& \left.+\sum_{q \neq l=0}^{L} \mathbb{E}\left(\tilde{A}_{l, n}^{N_{l}}(\varphi)\right) \mathbb{E}\left(\tilde{A}_{q, n}^{N_{q}}(\varphi)\right)\right)
\end{aligned}
$$

The bound therefore follows trivially from applying Theorems C.1 and Lemma C.2 from the Supplementary materials to each level.

REMARK 4.1. Observe that the bound of the first term in $B_{l}(n)$ of $(4.3)$ is limited by the coupled resampling, and is asymptotically proportional to $h_{l}^{\beta / 2}$, as proven in Theorem D.1. This is the reason for the reduced rate.

\section{Numerical Examples.}

5.1. Model Settings. The numerical performance of the MLPF algorithm will be illustrated here, with a few examples of the diffusion processes considered in this paper. Recall that the diffusions take the following form

$$
d X_{t}=a\left(X_{t}\right) d t+b\left(X_{t}\right) d W_{t}, \quad X_{0}=x_{0}
$$

with $X_{t} \in \mathbb{R}^{d}, t \geq 0$ and $\left\{W_{t}\right\}_{t \in[0, T]}$ a Brownian motion of appropriate dimension. In addition, partial observations $\left\{y_{1}, \ldots, y_{n}\right\}$ are available with $Y_{n}$ obtained at time $n \delta$, and $Y_{n} \mid X_{n \delta}$ has a density function $G\left(y_{n}, x_{n \delta}\right)$. The objective is the estimation of $\mathbb{E}\left[\varphi\left(X_{n \delta}\right) \mid y_{1: n}\right]$ for some test function $\varphi(x)$. Details of each example are described below. A summary of settings can be found in Table 5.1. Note that none of our examples satisfy Assumption 2.2 (i). However, that assumption is made only for technical convenience, in the sense that it significantly simplifies the calculations made for our main mathematical results, which are already quite lengthy. We believe that this assumption can be weakened to remove the lower bound on the likelihood, with an increase in the technical details in our proofs. The numerical results serve as a non-rigorous argument for this.

Ornstein-Uhlenbeck Process. First, consider the following OU process,

$$
\begin{gathered}
d X_{t}=\theta\left(\mu-X_{t}\right) d t+\sigma d W_{t}, \\
Y_{n} \mid X_{n \delta} \sim \mathcal{N}\left(X_{n \delta}, \tau^{2}\right), \quad \varphi(x)=x .
\end{gathered}
$$

An analytical solution exists for this process and the exact value of $\mathbb{E}\left[X_{n \delta} \mid y_{1: n}\right]$ can be computed using a Kalman filter. The constants in the example are, $x_{0}=0, \delta=0.5$, $\theta=1, \mu=0, \sigma=0.5$, and $\tau^{2}=0.2$.

Geometric Brownian Motion. Next consider the GBM process,

$$
\begin{gathered}
d X_{t}=\mu X_{t} d t+\sigma X_{t} d W_{t}, \\
Y_{n} \mid X_{n \delta} \sim \mathcal{N}\left(\log X_{n \delta}, \tau^{2}\right), \quad \varphi(x)=x,
\end{gathered}
$$

This process also admits an analytical solution, by using the transformation $Z_{t}=$ $\log X_{t}$. The constants are, $x_{0}=1, \delta=0.001, \mu=0.02, \sigma=0.2$ and $\tau^{2}=0.01$. 


\begin{tabular}{lllll}
\hline Example & $a(x)$ & $b(x)$ & $G(y ; x)$ & $\varphi(x)$ \\
\hline OU & $\theta(\mu-x)$ & $\sigma$ & $\mathcal{N}\left(x, \tau^{2}\right)$ & $x$ \\
GBM & $\mu x$ & $\sigma x$ & $\mathcal{N}\left(\log x, \tau^{2}\right)$ & $x$ \\
Langevin & $\frac{1}{2} \nabla \log \pi(x)$ & $\sigma$ & $\mathcal{N}\left(0, \tau^{2} e^{x}\right)$ & $\tau^{2} e^{x}$ \\
NLM & $\theta(\mu-x)$ & $\frac{\sigma}{\sqrt{1+x^{2}}}$ & $\mathcal{L}(x, s)$ & $x$ \\
\hline
\end{tabular}

Table 5.1: Model settings

Langevin Stochastic Differential Equation. Here the SDE is given by

$$
\begin{gathered}
d X_{t}=\frac{1}{2} \nabla \log \pi\left(X_{t}\right) d t+\sigma d W_{t}, \\
Y_{n} \mid X_{n \delta} \sim \mathcal{N}\left(0, \tau^{2} e^{X_{n \delta}}\right), \quad \varphi(x)=\tau^{2} e^{x}
\end{gathered}
$$

where $\pi(x)$ denotes a probability density function. The density $\pi(x)$ is chosen as the Student's $t$-distribution with degrees of freedom $\nu=10$. The other constants are, $x_{0}=0, \delta=1, \sigma=1$ and $\tau^{2}=1$. Real daily S\&P $500 \log$ return data (from August 3, 2011 to July 24, 2015, normalized to unity variance) is used.

An SDE with a Non-Linear Diffusion Term. Last, the following SDE is considered,

$$
\begin{gathered}
d X_{t}=\theta\left(\mu-X_{t}\right) d t+\frac{\sigma}{\sqrt{1+X_{t}^{2}}} d W_{t}, \\
Y_{n} \mid X_{n \delta} \sim \mathcal{L}\left(X_{n \delta}, s\right), \quad \varphi(x)=x,
\end{gathered}
$$

where $\mathcal{L}(m, s)$ denotes the Laplace distribution with location $m$ and scale $s$. The constants are $x_{0}=0, \delta=0.5, \theta=1, \mu=0, \sigma=1$ and $s=\sqrt{0.1}$. This example is abbreviated NLM in the remainder of this section.

5.2. Simulation Settings. For each example, multilevel estimators are considered at levels $L=1, \ldots, 8$. For the OU and GBM processes, the ground truth is computed through a Kalman filter. For the two other examples, results from particle filters at level $L=9$ are used as approximations to the ground truth.

For each level of MLPF algorithm, $N_{l}=\left\lfloor N_{0, L} h_{l}^{(\beta+2 \gamma) / 4}\right\rfloor$ particles are used, where $h_{l}=M_{l}^{-1}=2^{-l}$ is the width of the Euler-Maruyama discretization; $\gamma$ is the rate of computational cost, which is 1 for the examples considered here; and $\beta$ is the rate of the strong error. The value of $\beta$ is 2 if the diffusion term $b(x)$ is constant and 1 in general. The value $N_{0, L} \propto \varepsilon^{-2} K(\varepsilon)$ is set according to Table 4.1. For the cases in which the diffusion term is constant, we let $N_{0, L}=2^{2 L} L$, while for the other cases $N_{0, L}=2^{(9 / 4) L}$. Resampling is done adaptively. For the plain particle filters, resampling is done when ESS (effective sample size) is less than a quarter of the particle numbers. For the coupled filters, we use the ESS of the coarse filter as the measurement of discrepancy. Each simulation is repeated 100 times. Note that the analysis does not cover such stochastic resampling strategy, so this also serves as a numerical argument for extendability of the theory.

5.3. Results. First consider the rate $\beta / 2$ of the strong error. This rate can be estimated either by the sample variance of $\hat{\varphi}_{l}\left(X_{n \delta}\right)=\sum_{i=1}^{N_{l}}\left\{w_{1}^{l, i} \varphi\left(U_{n, 1}^{l, i}\right)-w_{2}^{l, i} \varphi\left(U_{n, 2}^{l, i}\right)\right\}$, or by $1-p_{l}(n)$, where $p_{l}(n)$ is the probability of the coupled particles having the same 


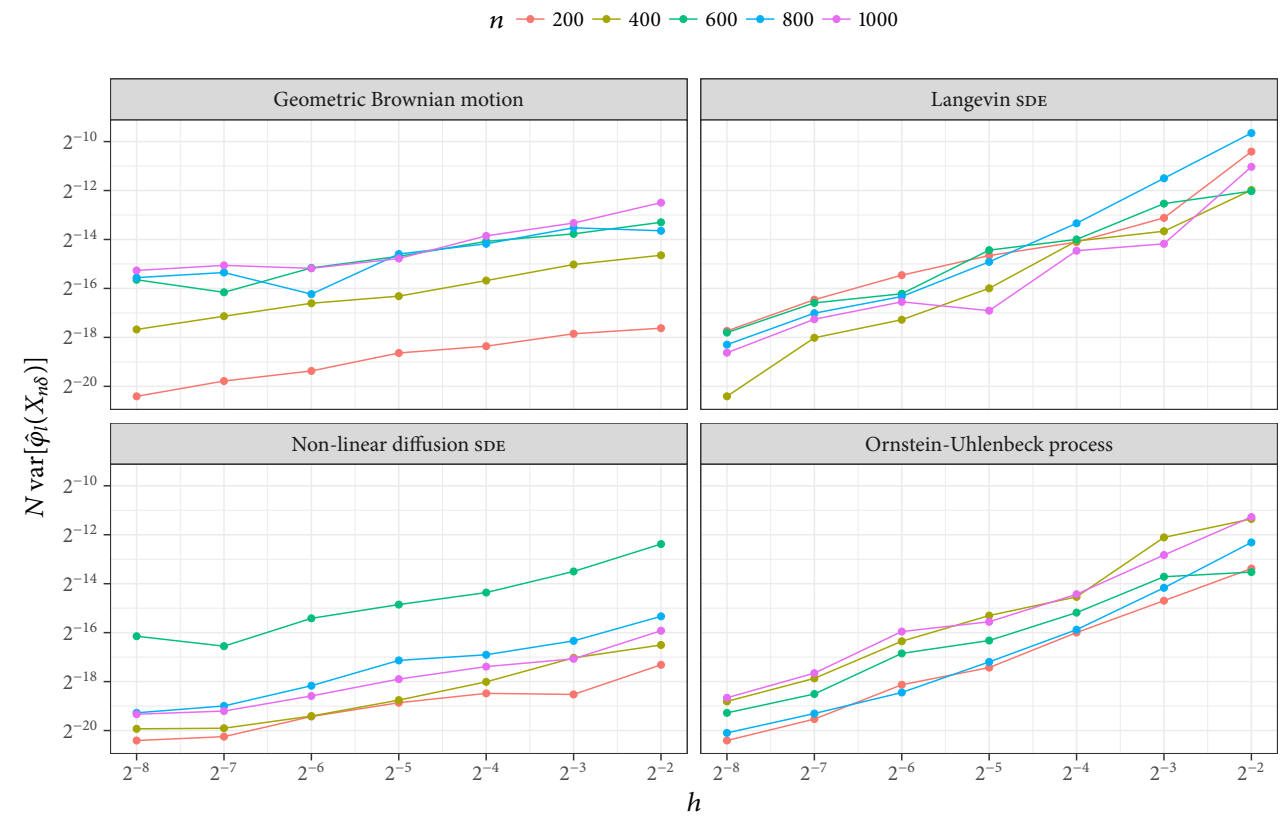

Fig. 5.1: Rate estimates using the variance.

\begin{tabular}{lrr}
\hline Example & PF & MLPF \\
\hline OU & -1.48 & -1.04 \\
GBM & -1.48 & -1.19 \\
Langevin & -1.46 & -1.14 \\
NLM & -1.49 & -1.24 \\
\hline
\end{tabular}

Table 5.2: Cost rate $\log \mathcal{C} \sim \log M S E$.

resampling index at time step $n$. Both $\operatorname{var}\left[\hat{\varphi}_{l}\left(X_{n \delta}\right)\right]$ and $p_{l}(n)$ can be estimated using the samples from MLPF simulations. Figures 5.1 and 5.2 show the estimated variance and value of $1-p_{l}(n)$ against $h_{l}$, respectively, scaled according to the minimum value for GBM, for comparison. The estimated rates for the OU and Langevin examples are about 1 . For the other two examples, where the diffusion term $b(x)$ is non-constant, the estimated rates are about 0.5. This is consistent with Corollary 4.1.

Next the rate of cost vs. MSE is examined. This is shown in Figure 5.3 and Table 5.2 for the estimator of $\mathbb{E}\left[\varphi\left(X_{n \delta}\right) \mid y_{1: n}\right]$. This agrees with the theory, which predicts a rate of -1.5 for the particle filter and a rate of -1.25 for the non-constant diffusion cases, and a logarithmic penalty on -1 for the others.

6. Conclusions. In this article a multilevel version of the particle filter has been introduced. The improvements that may be brought about by this approach were illustrated both theoretically and numerically. There are several natural extensions to this work. First, and perhaps most importantly, is to theoretically understand the 


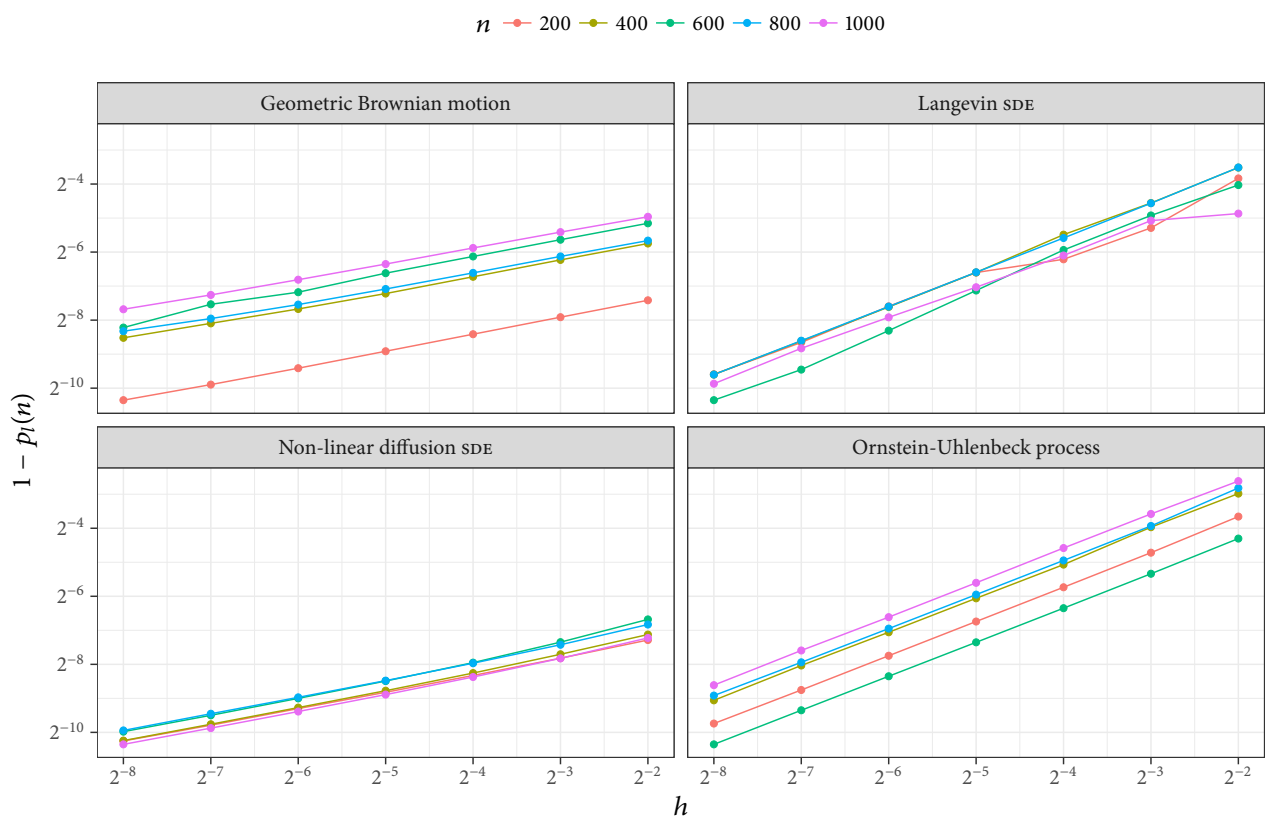

Fig. 5.2: Rate estimates using the probability of coupling.

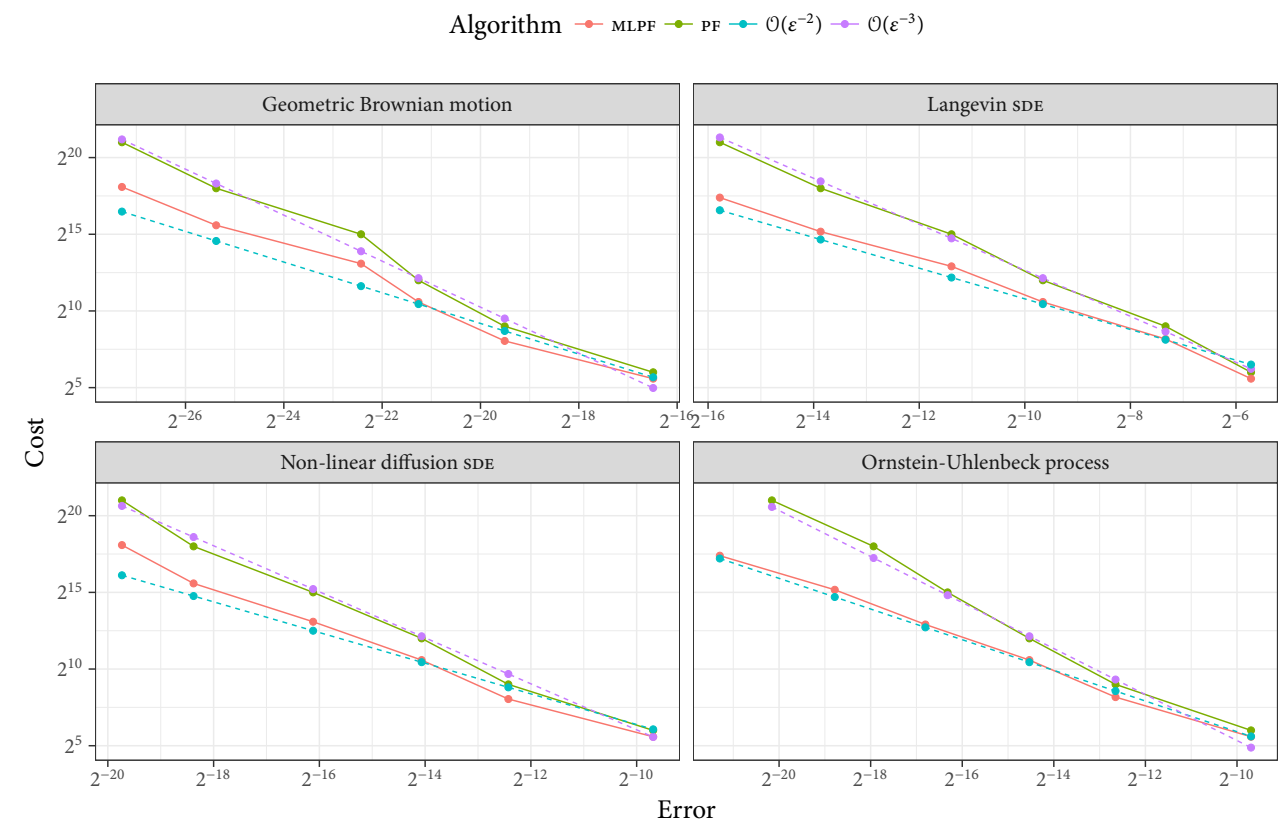

Fig. 5.3: Cost rates as a function of MSE. 
advantage of the particular coupled resampling mechanism adopted in this article, in comparison to other types of coupled resampling, e.g. via the variance in the CLT. It is remarked that other resampling strategies were tried on these examples, and they did not preserve a desired rate of strong convergence. However empirical results recently appeared in [18] which indicate that more favorable convergence rates may be preserved in certain cases by replacing the resampling step with a deterministic transformation. No proofs are found in this latter article, which has appeared around the same time as the first version of this paper [23]. Second, it would be of interest to explore techniques for improving the preservation of coupling such that the same MLMC rate $\beta$ carries through to the MLPF, rather than $\beta / 2$, e.g. via coupling the independent pairs of particle filters in some way, or perhaps through a different resampling strategy involving antithetic variables [15]. Strategies in [30], subsequent to the first version of this article [23], may achieve this, but at this time there is no proof of this. Finally, one can use the approach in e.g. [22] to improve the stability of the particle filtering algorithm.

Acknowledgements. We thank two referees for their comments which have greatly improved the paper. AJ, KL \& YZ were supported by an AcRF tier 2 grant: R-155-000-143-112. AJ is affiliated with the Risk Management Institute and the Center for Quantitative Finance at NUS. KL was supported by a Laboratory Directed Research and Development (LDRD) Strategic Hire grant from Oak Ridge National Laboratory (ORNL). KL \& AJ were additionally supported by King Abdullah University of Science and Technology (KAUST). KK was supported by CREST, JST and Grant-in-Aid for Young Scientists (B) 24740062.

\section{Appendix A. Set Up.}

A.1. Basic Notations. Consider a sequence of random variables $\left(v_{n}\right)_{n \geq 0}$ with $v_{n}=\left(u_{n, 1}, u_{n, 2}\right) \in \mathcal{U} \times \mathcal{U}=: \mathcal{V}$. For $\mu \in \mathcal{P}(\mathcal{V})$ (the probability measures on $\mathcal{V}$ ) and function $\varphi \in \mathcal{B}_{b}(\mathcal{U})$ (bounded-measurable, real-valued) we will write:

$$
\mu\left(\varphi_{j}\right)=\int_{\mathcal{V}} \varphi\left(u_{j}\right) \mu(d v) \quad j \in\{1,2\}, v=\left(u_{1}, u_{2}\right) .
$$

Write the $j \in\{1,2\}$ marginals (on $u_{j}$ ) of a probability $\mu \in \mathcal{P}(\mathcal{V})$ as $\mu_{j}$. Define the potentials: $G_{n}: \mathcal{U} \rightarrow \mathbb{R}_{+}$. Let $\eta_{0} \in \mathcal{P}(\mathcal{V})$ and define Markov kernels $M_{n}: \mathcal{V} \rightarrow \mathcal{P}(\mathcal{V})$ and $M_{n, j}: \mathcal{U} \rightarrow \mathcal{P}(\mathcal{U})$ with $n \geq 1$ and $j \in\{1,2\}$. It is explictly assumed that for $\varphi \in \mathcal{B}_{b}(\mathcal{U})$ the $j$ marginals satisfy:

$$
M_{n}\left(\varphi_{j}\right)(v)=\int_{\mathcal{V}} \varphi\left(u_{j}^{\prime}\right) M_{n}\left(v, d v^{\prime}\right)=\int_{\mathcal{U}} \varphi\left(u_{j}^{\prime}\right) M_{n, j}\left(u_{j}, d u_{j}^{\prime}\right) .
$$

We adopt the definition for $(v, \tilde{v})=\left(\left(u_{1}, u_{2}\right),\left(\tilde{u}_{1}, \tilde{u}_{2}\right)\right)$ of a sequence of Markov kernels $\left(\bar{M}_{n}\right)_{n \geq 1}, \bar{M}_{n}: \mathcal{V} \times \mathcal{V} \rightarrow \mathcal{P}(\mathcal{V})$

$$
\bar{M}_{n}\left((v, \tilde{v}), d v^{\prime}\right):=M_{n}\left(\left(u_{1}, \tilde{u}_{2}\right), d v^{\prime}\right) .
$$

In the main text $\mathcal{U}=\mathbb{R}^{d}$, and in the references that follow $\mathcal{U}$ should replace $\mathbb{R}^{d}$ in Assumptions 2.2 and 4.1 .

A.2. Marginal Feynman-Kac Formula. Given the above notations and defintions we define the $j$-marginal Feynman-Kac formulae:

$$
\gamma_{n, j}\left(d u_{n}\right)=\int \prod_{p=0}^{n-1} G_{p}\left(u_{p}\right) \eta_{0, j}\left(d u_{0}\right) \prod_{p=1}^{n} M_{p, j}\left(u_{p-1}, d u_{p}\right)
$$


with for $\varphi \in \mathcal{B}_{b}(\mathcal{U})$

$$
\eta_{n, j}(\varphi)=\frac{\gamma_{n, j}(\varphi)}{\gamma_{n, j}(1)}
$$

One can also define the sequence of Bayes operators, for $\mu \in \mathcal{P}(\mathcal{U})$

$$
\Phi_{n, j}(\mu)(d u)=\frac{\mu\left(G_{n-1} M_{n, j}(\cdot, d u)\right)}{\mu\left(G_{n-1}\right)} \quad n \geq 1 .
$$

Recall that for $n \geq 1, \eta_{n, j}=\Phi_{n, j}\left(\eta_{n-1, j}\right)$.

A.3. Feynman-Kac Formulae for Multi-Level Particle Filters. For $\mu \in$ $\mathcal{P}(\mathcal{V})$ define for $u \in \mathcal{U}, v \in \mathcal{V}:$

$$
\begin{aligned}
G_{n, j, \mu}(u) & =\frac{G_{n}(u)}{\mu_{j}\left(G_{n}\right)} \\
\bar{G}_{n, \mu}(v) & =G_{n, 1, \mu}\left(u_{1}\right) \wedge G_{n, 2, \mu}\left(u_{2}\right) .
\end{aligned}
$$

Now for any sequence $\left(\mu_{n}\right)_{n \geq 0}, \mu_{n} \in \mathcal{P}(\mathcal{V})$, define the sequence of operators $\left(\bar{\Phi}_{n}\left(\mu_{n-1}\right)\right)_{n \geq 1}$ :

$$
\begin{gathered}
\bar{\Phi}_{n}\left(\mu_{n-1}\right)\left(d v_{n}\right)= \\
\mu_{n-1}\left(\bar{G}_{n-1, \mu_{n-1}}\right) \frac{\mu_{n-1}\left(\bar{G}_{n-1, \mu_{n-1}} M_{n}\left(\cdot, d v_{n}\right)\right)}{\mu_{n-1}\left(\bar{G}_{n-1, \mu_{n-1}}\right)}+\left(1-\mu_{n-1}\left(\bar{G}_{n-1, \mu_{n-1}}\right)\right) \times \\
\mu_{n-1} \otimes \mu_{n-1}\left(\left[\frac{G_{n-1,1, \mu_{n-1}}-\bar{G}_{n-1, \mu_{n-1}}}{\mu_{n-1}\left(G_{n-1,1, \mu_{n-1}}-\bar{G}_{n-1, \mu_{n-1}}\right)} \otimes \frac{G_{n-1,2, \mu_{n-1}}-\bar{G}_{n-1, \mu_{n-1}}}{\mu_{n-1}\left(G_{n-1,2, \mu_{n-1}}-\bar{G}_{n-1, \mu_{n-1}}\right)}\right] \times\right. \\
\left.\bar{M}_{n}\left(\cdot, d v_{n}\right)\right)
\end{gathered}
$$

Now define $\bar{\eta}_{n}:=\bar{\Phi}_{n}\left(\bar{\eta}_{n-1}\right)$ for $n \geq 1, \bar{\eta}_{0}=\eta_{0}$.

Proposition A.1. Let $\left(\mu_{n}\right)_{n>0}$ be a sequence of probability measures on $\mathcal{V}$ with $\mu_{0}=\eta_{0}$ and for each $j \in\{1,2\}, \varphi \in \mathcal{B}_{b}(\mathcal{U})$

$$
\mu_{n}\left(\varphi_{j}\right)=\eta_{n, j}(\varphi) .
$$

Then:

$$
\eta_{n, j}(\varphi)=\bar{\Phi}_{n}\left(\mu_{n-1}\right)\left(\varphi_{j}\right) .
$$

In particular $\bar{\eta}_{n, j}=\eta_{n, j}$ for each $n \geq 0$.

Proof. By assumption $M_{n}\left(\varphi_{j}\right)=M_{n, j}(\varphi)$, so we have

$$
\begin{aligned}
\bar{\Phi}_{n}\left(\mu_{n-1}\right)\left(\varphi_{j}\right) & =\mu_{n-1}\left(\bar{G}_{n-1, \mu_{n-1}} M_{n, j}(\varphi)\right)+\mu_{n-1}\left(\left[G_{n-1, j, \mu_{n-1}}-\bar{G}_{n-1, \mu_{n-1}}\right] M_{n, j}(\varphi)\right) \\
& =\mu_{n-1}\left(G_{n-1, j, \mu_{n-1}} M_{n, j}(\varphi)\right) \\
& =\eta_{n-1, j}\left(G_{n-1, j, \mu_{n-1}} M_{n, j}(\varphi)\right) \\
& =\Phi_{n, j}\left(\eta_{n-1, j}\right)(\varphi) \\
& =\eta_{n, j}(\varphi) .
\end{aligned}
$$


REMARK A.1. It is established that for any $\mu \in \mathcal{P}(\mathcal{V})$

$$
\bar{\Phi}_{n}(\mu)\left(\varphi_{j}\right)=\Phi_{n, j}\left(\mu_{j}\right)(\varphi) .
$$

This property is very useful in subsequent calculations.

The point of the proposition is that if one has a system that samples $\bar{\eta}_{0}, \bar{\Phi}_{1}\left(\bar{\eta}_{0}\right)$ and so on, that marginally, one has exactly the marginals $\eta_{n, j}$ at each time point. In practice one cannot do this, but rather runs the following system:

$$
\left(\prod_{i=1}^{N} \bar{\eta}_{0}\left(d v_{0}^{i}\right)\right)\left(\prod_{p=1}^{n} \prod_{i=1}^{N} \bar{\Phi}_{p}\left(\bar{\eta}_{p-1}^{N}\right)\left(d v_{p}^{i}\right)\right)
$$

which is exactly one pair of particle filters at a given level of the MLPF.

\section{Appendix B. Normalizing Constant.}

First note that one can use the following

$$
\prod_{p=0}^{n-1} \bar{\eta}_{p, j}^{N}\left(G_{p}\right)
$$

to estimate $\gamma_{n, j}(1)$. It is now proven that this estimate is unbiased. More work on the MLPF for normalizing constant estimation can be found in [24].

In particular, it will be shown that

$$
\left(\prod_{p=0}^{n-1} \bar{\eta}_{p, j}^{N}\left(G_{p}\right)\right) \bar{\eta}_{n, j}^{N}(\varphi)
$$

is an unbiased estimator of $\gamma_{n, j}(\varphi)$, and the above follows immediately. The proof is by induction and the result at step 0 is clearly true. Now suppose it is true at step $n-1$ and consider the estimator above:

$$
\mathbb{E}\left[\left(\prod_{p=0}^{n-1} \bar{\eta}_{p, j}^{N}\left(G_{p}\right)\right) \bar{\eta}_{n, j}^{N}(\varphi) \mid \mathscr{F}_{n-1}^{N}\right]=\left(\prod_{p=0}^{n-1} \bar{\eta}_{p, j}^{N}\left(G_{p}\right)\right) \mathbb{E}\left[\bar{\eta}_{n, j}^{N}(\varphi) \mid \mathscr{F}_{n-1}^{N}\right]
$$

where $\mathscr{F}_{n-1}^{N}$ is the filtration generated by the particle system up-to time $n-1$. Now, by the exchangeability of the particle system and (A.2) :

$$
\mathbb{E}\left[\bar{\eta}_{n, j}^{N}(\varphi) \mid \mathscr{F}_{n-1}^{N}\right]=\bar{\Phi}_{n}\left(\bar{\eta}_{n-1}^{N}\right)\left(\varphi_{j}\right)=\Phi_{n, j}\left(\bar{\eta}_{n-1, j}^{N}\right)(\varphi)
$$

So

$$
\mathbb{E}\left[\left(\prod_{p=0}^{n-1} \bar{\eta}_{p, j}^{N}\left(G_{p}\right)\right) \bar{\eta}_{n, j}^{N}(\varphi)\right]=\mathbb{E}\left[\left(\prod_{p=0}^{n-2} \bar{\eta}_{p, j}^{N}\left(G_{p}\right)\right) \bar{\eta}_{n-1, j}^{N}\left(G_{n-1} M_{n, j}(\varphi)\right)\right] .
$$

The induction hypothesis and standard results complete the proof.

Appendix C. $\mathbb{L}_{2}$-Error.

The squared $\mathbb{L}_{2}$-Error (MSE) is considered here. 
C.1. Results for the Filter. Let

$B(n)=\left(\sum_{p=0}^{n} \mathbb{E}\left[\left\{\left|u_{p, 1}^{1}-u_{p, 2}^{1}\right| \wedge 1\right\}^{2}\right]^{1 / 2}+\left\|\eta_{p, 1}-\eta_{p, 2}\right\|_{\mathrm{tv}}+\sum_{p=1}^{n}\left\|\left|M_{p, 1}-M_{p, 2} \|\right|\right)^{2}\right.$.

Theorem C.1. Assume 2.2 and 4.1. Then for any $n \geq 0, \varphi \in \mathcal{B}_{b}(\mathcal{U}) \cap \operatorname{Lip}(\mathcal{U})$ there exist a $C(n, \varphi)<+\infty$ such that

$$
\mathbb{E}\left[\left(\frac{\bar{\eta}_{n}^{N}\left(G_{n, 1} \varphi_{1}\right)}{\bar{\eta}_{n}^{N}\left(G_{n, 1}\right)}-\frac{\bar{\eta}_{n}^{N}\left(G_{n, 2} \varphi_{2}\right)}{\bar{\eta}_{n}^{N}\left(G_{n, 2}\right)}-\frac{\bar{\eta}_{n}\left(G_{n, 1} \varphi_{1}\right)}{\bar{\eta}_{n}\left(G_{n, 1}\right)}+\frac{\bar{\eta}_{n}\left(G_{n, 2} \varphi_{2}\right)}{\bar{\eta}_{n}\left(G_{n, 2}\right)}\right)^{2}\right] \leq \frac{C(n, \varphi)}{N} B(n) .
$$

Proof. Follows directly from Lemma C.3 and similar calculations to the proof of Theorem C.2 for the term $\mathbb{E}\left[\left(\left[\bar{\Phi}_{n}\left(\bar{\eta}_{n-1}^{N}\right)-\bar{\eta}_{n}\right]\left(\varphi_{1}-\varphi_{2}\right)\right)^{2}\right]$.

Lemma C.1. Assume 2.2 and 4.1. Then for any $n \geq 1, \varphi \in \mathcal{B}_{b}(\mathcal{U})$ there exist a $C(n, \varphi)<+\infty$ such that

$$
\left|\mathbb{E}\left[\frac{\bar{\eta}_{n}^{N}\left(G_{n, 1} \varphi_{1}\right)}{\bar{\eta}_{n}^{N}\left(G_{n, 1}\right)}-\frac{\bar{\eta}_{n}\left(G_{n, 1} \varphi_{1}\right)}{\bar{\eta}_{n}\left(G_{n, 1}\right)}\right]\right|+\left|\mathbb{E}\left[\frac{\bar{\eta}_{n}^{N}\left(G_{n, 2} \varphi_{2}\right)}{\bar{\eta}_{n}^{N}\left(G_{n, 2}\right)}-\frac{\bar{\eta}_{n}\left(G_{n, 2} \varphi_{2}\right)}{\bar{\eta}_{n}\left(G_{n, 2}\right)}\right]\right| \leq \frac{C(n, \varphi)}{N} .
$$

Proof. The proof follows by using the bias result of Proposition 9.5.6 of [8] (which holds in our context, see also Proposition C.1). $\square$

Lemma C.2. Assume 2.2 and 4.1. Then for any $n \geq 1, \varphi \in \mathcal{B}_{b}(\mathcal{U})$ there exist a $C(n, \varphi)<+\infty$ such that

$$
\left|\mathbb{E}\left[\frac{\bar{\eta}_{n}^{N}\left(G_{n, 1} \varphi_{1}\right)}{\bar{\eta}_{n}^{N}\left(G_{n, 1}\right)}-\frac{\bar{\eta}_{n}^{N}\left(G_{n, 2} \varphi_{2}\right)}{\bar{\eta}_{n}^{N}\left(G_{n, 2}\right)}-\frac{\bar{\eta}_{n}\left(G_{n, 1} \varphi_{1}\right)}{\bar{\eta}_{n}\left(G_{n, 1}\right)}+\frac{\bar{\eta}_{n}\left(G_{n, 2} \varphi_{2}\right)}{\bar{\eta}_{n}\left(G_{n, 2}\right)}\right]\right| \leq C(n, \varphi) \frac{\sqrt{B(n)}}{N} .
$$

Proof. For $p \leq n$ and for $j=1,2$, let

$$
Q_{p, n, j}(\varphi)\left(v_{p}\right)=\int G_{n}\left(u_{n, j}\right) \varphi\left(u_{n, j}\right) \prod_{p \leq q<n} G_{q}\left(u_{q, j}\right) M_{q, j}\left(u_{q, j}, d u_{q+1, j}\right)\left(v_{p}=\left(u_{p, 1}, u_{p, 2}\right)\right) .
$$

Observe that

$$
\begin{aligned}
\bar{\eta}_{p}\left(Q_{p, n, 1}(\varphi)\right)-\bar{\eta}_{p}\left(Q_{p, n, 2}(\varphi)\right) & =\mathcal{O}\left(\left\|\eta_{p, 1}-\eta_{p, 2}\right\|_{\mathrm{tv}}+\sum_{p \leq q<n}\left\|\mid M_{p, 1}-M_{p, 2}\right\|\right) \\
& =\mathcal{O}(\sqrt{B(n)}) .
\end{aligned}
$$

We prove the following by induction on $p \leq n$ :

$$
\left|\mathbb{E}\left[\left(\bar{\eta}_{p}^{N}-\bar{\eta}_{p}\right)\left(Q_{p, n, 1}(\varphi)-Q_{p, n, 2}(\varphi)\right)\right]\right| \leq C(n, \varphi) \frac{\sqrt{B(n)}}{N} .
$$

The expectation is 0 for $p=0$ by definition. Note that

$$
\begin{aligned}
\mathbb{E}\left[\left(\bar{\eta}_{p+1}^{N}-\bar{\eta}_{p+1}\right)\left(Q_{p+1, n, 1}(\varphi)-Q_{p+1, n, 2}(\varphi)\right)\right] & =\mathbb{E}\left[( \overline { \Phi } _ { p + 1 } ( \overline { \eta } _ { p } ^ { N } ) - \overline { \eta } _ { p + 1 } ) \left(Q_{p+1, n, 1}(\varphi)-\right.\right. \\
& \left.\left.Q_{p+1, n, 2}(\varphi)\right)\right] \\
& =\mathbb{E}\left[\frac{\bar{\eta}_{p}^{N}\left(Q_{p, n, 1}(\varphi)\right)}{\bar{\eta}_{p}^{N}\left(G_{p, 1}\right)}-\frac{\bar{\eta}_{p}^{N}\left(Q_{p, n, 2}(\varphi)\right)}{\bar{\eta}_{p}^{N}\left(G_{p, 2}\right)}\right. \\
& \left.-\frac{\bar{\eta}_{p}\left(Q_{p, n, 1}(\varphi)\right)}{\bar{\eta}_{p}\left(G_{p, 1}\right)}+\frac{\bar{\eta}_{p}\left(Q_{p, n, 2}(\varphi)\right)}{\bar{\eta}_{p}\left(G_{p, 2}\right)}\right] .
\end{aligned}
$$


Thus by taking $p=n$, the proof is complete if we can show (C.3). To prove (C.3), the departure point is Lemma C.3, letting $a=\bar{\eta}_{p}^{N}\left(Q_{p, n, 1}(\varphi)\right), A=\bar{\eta}_{p}^{N}\left(G_{p, 1}\right), b=$ $\bar{\eta}_{p}^{N}\left(Q_{p, n, 2}(\varphi)\right), B=\bar{\eta}_{p}^{N}\left(G_{p, 2}\right), c=\bar{\eta}_{p}\left(Q_{p, n, 1}(\varphi)\right), C=\bar{\eta}_{p}\left(G_{p, 1}\right), d=\bar{\eta}_{p}\left(Q_{p, n, 2}(\varphi)\right)$, and $D=\bar{\eta}_{p}\left(G_{p, 2}\right)$. Note the following estimates hold, by Theorem 3.1 of [11]

$$
\mathbb{E}\left[|a-c|^{2}\right]^{1 / 2}, \mathbb{E}\left[|b-d|^{2}\right]^{1 / 2}, \mathbb{E}\left[|A-C|^{2}\right]^{1 / 2}, \mathbb{E}\left[|B-D|^{2}\right]^{1 / 2}=\mathcal{O}\left(N^{-1 / 2}\right),
$$

as well as the following, by Lemma C.1

$$
\mathbb{E}[a]-c, \mathbb{E}[b]-d, \mathbb{E}[A]-C, \mathbb{E}[B]-D=\mathcal{O}\left(N^{-1}\right) .
$$

Also, by (C.2),

$$
c-d, C-D=\mathcal{O}(\sqrt{B(n)}) .
$$

Hence, by Equations (C.6) and (C.5) (noting that $c, C, d, D$ are not random), the last 4 terms of Lemma C.3 are bounded by $\frac{C(n, \varphi)}{N} \sqrt{B(n)}$.

Now, note that the first two terms of Lemma C.3 can be further decomposed into

$$
\begin{aligned}
\mathbb{E}\left[\frac{a-b-(c-d)}{A}-\frac{b[A-B-(C-D)]}{A B}\right] & =\frac{\mathbb{E}[a-b-(c-d)]}{C}-\frac{d \mathbb{E}[A-B-(C-D)]}{C D} \\
& -\mathbb{E}\left[\frac{(A-C)[a-b-(c-d)]}{A C}\right] \\
& -\mathbb{E}[[A-B-(C-D)] \times \\
& \left.\frac{(C-A) D b+(D-B) A b+(b-d) A B}{A B C D}\right] .
\end{aligned}
$$

The last two expectations above were $\mathcal{O}(1 / N)$ by applying Cauchy-Schwartz inequality and using (C.4). Now, the first two terms above will be dealt with using the inductive hypothesis. Hence the proof is complete.

口

C.2. Results for the Predictor. Theorem C.2. Assume 2.2 and 4.1. Then for any $n \geq 0, \varphi \in \mathcal{B}_{b}(\mathcal{U}) \cap \operatorname{Lip}(\mathcal{U})$ there exist a $C(n, \varphi)<+\infty$ such that

$$
\mathbb{E}\left[\left(\left[\bar{\eta}_{n}^{N}-\bar{\eta}_{n}\right]\left(\varphi_{1}-\varphi_{2}\right)\right)^{2}\right] \leq \frac{C(n, \varphi)}{N} B(n) .
$$

Proof. The proof is by induction and clearly holds at step 0 by the MarcinkiewiczZygmund inequality (see e.g. [5]) so we proceed to the induction step. Throughout $C$ is a constant whose value may change from line-to-line. Any important dependencies are given a function notation.

$$
\begin{gathered}
\mathbb{E}\left[\left(\left[\bar{\eta}_{n}^{N}-\bar{\eta}_{n}\right]\left(\varphi_{1}-\varphi_{2}\right)\right)^{2}\right] \leq \\
2 \mathbb{E}\left[\left(\left[\bar{\eta}_{n}^{N}-\bar{\Phi}_{n}\left(\bar{\eta}_{n-1}^{N}\right)\right]\left(\varphi_{1}-\varphi_{2}\right)\right)^{2}\right]+2 \mathbb{E}\left[\left(\left[\bar{\Phi}_{n}\left(\bar{\eta}_{n-1}^{N}\right)-\bar{\eta}_{n}\right]\left(\varphi_{1}-\varphi_{2}\right)\right)^{2}\right] .
\end{gathered}
$$

Consider the two terms on the R.H.S. of (C.7) separately. 
Term: $\mathbb{E}\left[\left(\left[\bar{\eta}_{n}^{N}-\bar{\Phi}_{n}\left(\bar{\eta}_{n-1}^{N}\right)\right]\left(\varphi_{1}-\varphi_{2}\right)\right)^{2}\right]$.

Begin by conditioning on $\mathscr{F}_{n-1}^{N}$ and then apply the Marcinkiewicz-Zygmund inequality to yield that

$$
\begin{gathered}
\mathbb{E}\left[\left(\left[\bar{\eta}_{n}^{N}-\bar{\Phi}_{n}\left(\bar{\eta}_{n-1}^{N}\right)\right]\left(\varphi_{1}-\varphi_{2}\right)\right)^{2}\right] \leq \\
\frac{C}{N}\left(\mathbb{E}\left[\left|\varphi\left(u_{n, 1}^{1}\right)-\varphi\left(u_{n, 2}^{1}\right)\right|^{2}\right]+\mathbb{E}\left[\left|\bar{\Phi}_{n}\left(\bar{\eta}_{n-1}^{N}\right)\left(\varphi_{1}-\varphi_{2}\right)\right|^{2}\right]\right) \leq \\
\frac{C}{N}\left(\mathbb{E}\left[\left\{\left|u_{n, 1}^{1}-u_{n, 2}^{1}\right| \wedge 1\right\}^{2}\right]+\mathbb{E}\left[\left|\bar{\Phi}_{n}\left(\bar{\eta}_{n-1}^{N}\right)\left(\varphi_{1}-\varphi_{2}\right)\right|^{2}\right]\right)
\end{gathered}
$$

where the final line follows since $\varphi \in \mathcal{B}_{b}(\mathcal{U}) \cap \operatorname{Lip}(\mathcal{U})$.

Now by (A.2)

$$
\begin{aligned}
\bar{\Phi}_{n}\left(\bar{\eta}_{n-1}^{N}\right)\left(\varphi_{1}-\varphi_{2}\right)= & \frac{\eta_{n-1,1}^{N}\left(G_{n-1} M_{n, 1}(\varphi)\right)-\eta_{n-1,2}^{N}\left(G_{n-1} M_{n, 2}(\varphi)\right)}{\eta_{n-1,1}^{N}\left(G_{n-1}\right)}+ \\
& \frac{\eta_{n-1,2}^{N}\left(G_{n-1} M_{n, 2}(\varphi)\right)}{\eta_{n-1,1}^{N}\left(G_{n-1}\right) \eta_{n-1,2}^{N}\left(G_{n-1}\right)}\left[\eta_{n-1,2}^{N}\left(G_{n-1}\right)-\eta_{n-1,1}^{N}\left(G_{n-(1)}(\mathbf{C}) .9\right)\right.
\end{aligned}
$$

Consider the first term on the R.H.S. of (C.9).

$$
\begin{gathered}
\frac{\eta_{n-1,1}^{N}\left(G_{n-1} M_{n, 1}(\varphi)\right)-\eta_{n-1,2}^{N}\left(G_{n-1} M_{n, 2}(\varphi)\right)}{\eta_{n-1,1}^{N}\left(G_{n-1}\right)}=\eta_{n-1,1}^{N}\left(G_{n-1}\right)^{-1}\left[\eta_{n-1,1}^{N}\left(G_{n-1} M_{n, 1}(\varphi)\right)\right. \\
\left.-\eta_{n-1,1}^{N}\left(G_{n-1} M_{n, 2}(\varphi)\right)+\eta_{n-1,1}^{N}\left(G_{n-1} M_{n, 2}(\varphi)\right)-\eta_{n-1,2}^{N}\left(G_{n-1} M_{n, 2}(\varphi)\right)\right] \quad \text { (C.10) }
\end{gathered}
$$

Now we deal with $\eta_{n-1,1}^{N}\left(G_{n-1} M_{n, 2}(\varphi)\right)-\eta_{n-1,2}^{N}\left(G_{n-1} M_{n, 2}(\varphi)\right)$ on the R.H.S. of (C.10).

$$
\begin{gathered}
\eta_{n-1,1}^{N}\left(G_{n-1} M_{n, 2}(\varphi)\right)-\eta_{n-1,2}^{N}\left(G_{n-1} M_{n, 2}(\varphi)\right)= \\
\frac{1}{N} \sum_{i=1}^{N}\left\{\left[G_{n-1}\left(u_{n-1,1}^{i}\right)-G_{n-1}\left(u_{n-1,2}^{i}\right)\right] M_{n, 2}(\varphi)\left(u_{n-1,1}^{i}\right)+\right. \\
\left.G_{n-1}\left(u_{n-1,2}^{i}\right)\left[M_{n, 2}(\varphi)\left(u_{n-1,1}^{i}\right)-M_{n, 2}(\varphi)\left(u_{n-1,2}^{i}\right)\right]\right\} .
\end{gathered}
$$

Then applying Assumptions 2.2 and 4.1 it follows that

$$
\left|\eta_{n-1,1}^{N}\left(G_{n-1} M_{n, 2}(\varphi)\right)-\eta_{n-1,2}^{N}\left(G_{n-1} M_{n, 2}(\varphi)\right)\right| \leq C(\varphi) \frac{1}{N} \sum_{i=1}^{N}\left\{\left|u_{n-1,1}^{i}-u_{n-1,2}^{i}\right| \wedge 1\right\}
$$

Returning to (C.10) it follows that

$$
\left|\eta_{n-1,1}^{N}\left(G_{n-1} M_{n, 1}(\varphi)\right)-\eta_{n-1,1}^{N}\left(G_{n-1} M_{n, 2}(\varphi)\right)\right| \leq C(\varphi)||\left|M_{n, 1}-M_{n, 2}\right||| .
$$


Thus using Assumptions 2.2 and 4.1 and noting (C.11)

$$
\begin{gathered}
\frac{\eta_{n-1,1}^{N}\left(G_{n-1} M_{n, 1}(\varphi)\right)-\eta_{n-1,2}^{N}\left(G_{n-1} M_{n, 2}(\varphi)\right)}{\eta_{n-1,1}^{N}\left(G_{n-1}\right)} \leq \\
C(\varphi)\left(\frac{1}{N} \sum_{i=1}^{N}\left\{\left|u_{n-1,1}^{i}-u_{n-1,2}^{i}\right| \wedge 1\right\}+||\left|M_{n, 1}-M_{n, 2}\right|||\right) .
\end{gathered}
$$

Returning to (C.9) and the second term on the R.H.S. it follows by the Lipschitz property of $G_{n-1}$ and the upper-bound on $\varphi$ and lower bound on $G_{n-1}$ that

$$
\begin{gathered}
\frac{\eta_{n-1,2}^{N}\left(G_{n-1} M_{n, 2}(\varphi)\right)}{\eta_{n-1,1}^{N}\left(G_{n-1}\right) \eta_{n-1,2}^{N}\left(G_{n-1}\right)}\left[\eta_{n-1,2}^{N}\left(G_{n-1}\right)-\eta_{n-1,1}^{N}\left(G_{n-1}\right)\right] \leq \\
C(\varphi) \frac{1}{N} \sum_{i=1}^{N}\left\{\left|u_{n-1,1}^{i}-u_{n-1,2}^{i}\right| \wedge 1\right\}
\end{gathered}
$$

Recalling (C.9) and noting (C.12)-(C.13)

$$
\bar{\Phi}_{n}\left(\bar{\eta}_{n-1}^{N}\right)\left(\varphi_{1}-\varphi_{2}\right) \leq C(\varphi)\left(\frac{1}{N} \sum_{i=1}^{N}\left\{\left|u_{n-1,1}^{i}-u_{n-1,2}^{i}\right| \wedge 1\right\}+||\left|M_{n, 1}-M_{n, 2}\right|||\right) .
$$

Thus, on returning to (C.8) it follows that

$$
\begin{gathered}
\mathbb{E}\left[\left(\left[\bar{\eta}_{n}^{N}-\bar{\Phi}_{n}\left(\bar{\eta}_{n-1}^{N}\right)\right]\left(\varphi_{1}-\varphi_{2}\right)\right)^{2}\right] \leq \\
\frac{C(\varphi)}{N}\left(\mathbb{E}\left[\left\{\left|u_{n-1,1}^{i}-u_{n-1,2}^{i}\right| \wedge 1\right\}^{2}\right]+\mathbb{E}\left[\left(\frac{1}{N} \sum_{i=1}^{N}\left\{\left|u_{n-1,1}^{i}-u_{n-1,2}^{i}\right| \wedge 1\right\}+||\left|M_{n, 1}-M_{n, 2}\right|||\right)^{2}\right]\right) \leq \\
\frac{C(\varphi)}{N}\left(\mathbb{E}\left[\left\{\left|u_{n-1,1}^{i}-u_{n-1,2}^{i}\right| \wedge 1\right\}^{2}\right]+\mathbb{E}\left[\left\{\left|u_{n-1,1}^{i}-u_{n-1,2}^{i}\right| \wedge 1\right\}^{2}\right]+\left.||\left|M_{n, 1}-M_{n, 2}\right|\right|^{2}\right) .
\end{gathered}
$$

The final equation follows from Jensen's inequality.

Term: $\mathbb{E}\left[\left(\left[\bar{\Phi}_{n}\left(\bar{\eta}_{n-1}^{N}\right)-\bar{\eta}_{n}\right]\left(\varphi_{1}-\varphi_{2}\right)\right)^{2}\right]$.

Application of Lemma C.3 to $\left[\bar{\Phi}_{n}\left(\bar{\eta}_{n-1}^{N}\right)-\bar{\eta}_{n}\right]\left(\varphi_{1}-\varphi_{2}\right)$ allows one to treat the six terms independently, by the $C_{2}$-inequality. Denote the upper-bound in the induction hypothesis at time $n-1$ as $B_{n-1}(N)$ (omitting dependence on the function), to avoid complex notations.

Term 1: First

$$
\begin{gathered}
\mathbb{E}\left[\left(\frac { 1 } { \eta _ { n - 1 , 1 } ^ { N } ( G _ { n - 1 } ) } \left(\eta_{n-1,1}^{N}\left(G_{n-1} M_{n, 1}(\varphi)\right)-\eta_{n-1,2}^{N}\left(G_{n-1} M_{n, 2}(\varphi)\right)-\right.\right.\right. \\
\left.\left.\left.\eta_{n-1,1}\left(G_{n-1} M_{n, 1}(\varphi)\right)+\eta_{n-1,2}\left(G_{n-1} M_{n, 2}(\varphi)\right)\right)\right)^{2}\right] \leq
\end{gathered}
$$




$$
\begin{gathered}
C \mathbb{E}\left[\left(\eta_{n-1,1}^{N}\left(G_{n-1} M_{n, 1}(\varphi)-G_{n-1} M_{n, 2}(\varphi)\right)-\eta_{n-1,1}\left(G_{n-1} M_{n, 1}(\varphi)-G_{n-1} M_{n, 2}(\varphi)\right)\right)^{2}\right]+ \\
\left.\left.\mathbb{E}\left[\left(\left[\bar{\eta}_{n-1}^{N}-\bar{\eta}_{n-1}\right]\left(\left[G_{n-1} M_{n, 2}(\varphi)\right)\right]_{1}-\left[G_{n-1} M_{n, 2}(\varphi)\right)\right]_{2}\right)\right)^{2}\right] .
\end{gathered}
$$

Application of Proposition C.1 and the induction hypothesis yields the upper bound:

$$
\frac{C(n)\left\|\left|M_{n, 1}-M_{n, 2}\right|\right\| \mid}{N}+B_{n-1}(N) .
$$

Term 2:

$$
\begin{gathered}
\mathbb{E}\left[\left(\frac { \eta _ { n - 1 , 2 } ^ { N } ( G _ { n - 1 } M _ { n , 2 } ( \varphi ) ) } { \eta _ { n - 1 , 1 } ^ { N } ( G _ { n - 1 } ) \eta _ { n - 1 , 2 } ^ { N } ( G _ { n - 1 } ) } \left(\eta_{n-1,1}^{N}\left(G_{n-1}\right)-\eta_{n-1,1}\left(G_{n-1}\right)-\right.\right.\right. \\
\left.\left.\eta_{n-1,2}^{N}\left(G_{n-1}\right)+\eta_{n-1,2}\left(G_{n-1}\right)\right)\right) \\
\leq C B_{n-1}(N) .
\end{gathered}
$$

Term 3: By Proposition C.1

$$
\begin{gathered}
\mathbb{E}\left[\left(\frac { 1 } { \eta _ { n - 1 , 1 } ^ { N } ( G _ { n - 1 } ) \eta _ { n - 1 , 1 } ( G _ { n - 1 } ) } ( \eta _ { n - 1 , 1 } - \eta _ { n - 1 , 1 } ^ { N } ) ( G _ { n - 1 } ) \left(\eta_{n-1,1}\left(G_{n-1} M_{n, 1}(\varphi)\right)-\right.\right.\right. \\
\left.\left.\left.\eta_{n-1,2}\left(G_{n-1} M_{n, 2}(\varphi)\right)\right)\right)^{2}\right] \leq
\end{gathered}
$$

$\frac{C(n)}{N}\left(||\left|M_{n, 1}-M_{n, 1}\right|\left\|^{2}+\right\| \eta_{n-1,1}-\eta_{n-1,2}\left\|_{\mathrm{tv}}^{2}+||\left|M_{n, 1}-M_{n, 1}\right|||\right\| \eta_{n-1,1}-\eta_{n-1,2} \|_{\mathrm{tv}}\right)$.

Term 4: By Proposition C.1

$$
\begin{gathered}
\mathbb{E}\left[\left(\frac{1}{\eta_{n-1,1}^{N}\left(G_{n-1}\right) \eta_{n-1,2}^{N}\left(G_{n-1}\right)}\left(\eta_{n-1,2}^{N}\left(G_{n-1} M_{n, 2}(\varphi)\right)-\eta_{n-1,2}\left(G_{n-1} M_{n, 2}(\varphi)\right)\right)\right.\right. \\
\left.\left.\left(\eta_{n-1,1}\left(G_{n-1}\right)-\eta_{n-1,2}\left(G_{n-1}\right)\right)\right)^{2}\right] \leq \frac{C(n)}{N}\left\|\eta_{n-1,1}-\eta_{n-1,2}\right\|_{\mathrm{tv}}^{2}
\end{gathered}
$$

Term 5: By Proposition C.1

$$
\begin{gathered}
\mathbb{E}\left[\left(\frac{\eta_{n-1,2}\left(G_{n-1} M_{n, 2}(\varphi)\right)}{\eta_{n-1,1}\left(G_{n-1}\right) \eta_{n-1,2}^{N}\left(G_{n-1}\right) \eta_{n-1,2}\left(G_{n-1}\right)}\left(\eta_{n-1,2}^{N}\left(G_{n-1}\right)-\eta_{n-1,2}\left(G_{n-1}\right)\right)\right.\right. \\
\left.\left.\left(\eta_{n-1,1}\left(G_{n-1}\right)-\eta_{n-1,2}\left(G_{n-1}\right)\right)\right)^{2}\right] \leq \frac{C(n)}{N}\left\|\eta_{n-1,1}-\eta_{n-1,2}\right\|_{\mathrm{tv}}^{2}
\end{gathered}
$$

Term 6: By Proposition C.1

$$
\mathbb{E}\left[\left(\frac{\eta_{n-1,2}\left(G_{n-1} M_{n, 2}(\varphi)\right)}{\eta_{n-1,1}^{N}\left(G_{n-1}\right) \eta_{n-1,1}\left(G_{n-1}\right) \eta_{n-1,2}^{N}\left(G_{n-1}\right)}\left(\eta_{n-1,1}^{N}\left(G_{n-1}\right)-\eta_{n-1,1}\left(G_{n-1}\right)\right)\right.\right.
$$




$$
\left.\left.\left(\eta_{n-1,1}\left(G_{n-1}\right)-\eta_{n-1,2}\left(G_{n-1}\right)\right)\right)^{2}\right] \leq \frac{C(n)}{N}\left\|\eta_{n-1,1}-\eta_{n-1,2}\right\|_{\mathrm{tv}}^{2} .
$$

Putting together the bounds on the terms 1-6 along with the bound on $\mathbb{E}\left[\left(\left[\bar{\eta}_{n}^{N}-\right.\right.\right.$ $\left.\left.\left.\bar{\Phi}_{n}\left(\bar{\eta}_{n-1}^{N}\right)\right]\left(\varphi_{1}-\varphi_{2}\right)\right)^{2}\right]$ completes the proof.

Lemma C.3. Let $a, b, c, d, A, B, C, D \in \mathbb{R}$ with $A, B, C, D$ non-zero then:

$$
\begin{gathered}
\frac{a}{A}-\frac{b}{B}-\left(\frac{c}{C}-\frac{d}{D}\right)=\frac{[a-b-(c-d)]}{A}-\frac{b[A-B-(C-D)]}{A B}+\frac{1}{A C}[C-A][c-d] \\
-\frac{1}{A B}(b-d)(C-D)+\frac{d}{C B D}(B-D)(C-D)+\frac{d}{A C B}(A-C)(C-D) .
\end{gathered}
$$

Proposition C.1. Assume 2.2 and 4.1. Then for any $n \geq 0, p \geq 1$ there exists a $C(n, p)<+\infty$ such that for any $\varphi \in \mathcal{B}_{b}(\mathcal{U}), j \in\{1,2\}$,

$$
\mathbb{E}\left[\left|\left[\eta_{n, j}^{N}-\eta_{n, j}\right](\varphi)\right|^{p}\right]^{1 / p} \leq \frac{C(n, p)\|\varphi\|}{\sqrt{N}} .
$$

Proof. The proof is by induction and clearly holds at rank 0 by the MarcinkiewiczZygmund inequality so we proceed to the induction step. Throughout $C$ is a constant whose value may change from line-to-line. Any important dependencies are given a function notation.

The triangle inequality provides

$\mathbb{E}\left[\left|\left[\eta_{n, j}^{N}-\eta_{n, j}\right](\varphi)\right|^{p}\right]^{1 / p} \leq \mathbb{E}\left[\left|\eta_{n, j}^{N}(\varphi)-\bar{\Phi}_{n}\left(\bar{\eta}_{n-1}^{N}\right)\left(\varphi_{j}\right)\right|^{p}\right]^{1 / p}+\mathbb{E}\left[\left|\bar{\Phi}_{n}\left(\bar{\eta}_{n-1}^{N}\right)\left(\varphi_{j}\right)-\eta_{n, j}(\varphi)\right|^{p}\right]^{1 / p}$.

For the first term on the R.H.S. one can condition on $\mathscr{F}_{n-1}^{N}$ and then apply the Marcinkiewicz-Zygmund inequality to yield that

$$
\mathbb{E}\left[\left|\eta_{n, j}^{N}(\varphi)-\bar{\Phi}_{n}\left(\bar{\eta}_{n-1}^{N}\right)\left(\varphi_{j}\right)\right|^{p}\right]^{1 / p} \leq \frac{C(n, p)\|\varphi\|}{\sqrt{N}} .
$$

For the second term on the R.H.S. one has the decomposition (see (A.2))

$$
\begin{gathered}
\bar{\Phi}_{n}\left(\bar{\eta}_{n-1}^{N}\right)\left(\varphi_{j}\right)-\eta_{n, j}(\varphi)= \\
\eta_{n-1, j}^{N}\left(G_{n-1}\right)^{-1}\left[\eta_{n-1, j}^{N}\left(G_{n-1} M_{n, j}(\varphi)\right)-\eta_{n-1, j}\left(G_{n-1} M_{n, j}(\varphi)\right)\right]+ \\
\frac{\eta_{n-1, j}\left(G_{n-1} M_{n, j}(\varphi)\right)}{\eta_{n-1, j}^{N}\left(G_{n-1}\right) \eta_{n-1, j}\left(G_{n-1}\right)}\left[\eta_{n-1, j}\left(G_{n-1}\right)-\eta_{n-1, j}^{N}\left(G_{n-1}\right)\right] .
\end{gathered}
$$

Then one can control $\mathbb{E}\left[\left|\bar{\Phi}_{n}\left(\bar{\eta}_{n-1}^{N}\right)\left(\varphi_{j}\right)-\eta_{n, j}(\varphi)\right|^{p}\right]^{1 / p}$ via Minkowski, Assumptions 2.2 and 4.1 and the induction hypothesis, to yield

$$
\mathbb{E}\left[\left|\bar{\Phi}_{n}\left(\bar{\eta}_{n-1}^{N}\right)\left(\varphi_{j}\right)-\eta_{n, j}(\varphi)\right|^{p}\right]^{1 / p} \leq \frac{C(n, p)\|\varphi\|}{\sqrt{N}},
$$


and this allows one to conclude.

Appendix D. Estimates for Stochastic Diffusion Processes.

Consider the case of the diffusion example (2.1) of Section 2, with the multilevel kernel introduced in Subsection 3.1. Fix a level $l$, and for $x, y \in \mathbb{R}^{d}$, let $\left(X_{1}^{x}, X_{2}^{y}\right) \sim$ $M((x, y)$, . $)$ be correlated Euler-Maruyama scheme of the solution process of the stochastic differential equation (2.1) i.e. $X_{1}^{x}$ is the solution at step $2^{l}$ of equation (2.3) with initial condition $x$ and $X_{2}^{y}$ is the solution at step $2^{l-1}$ of equation (3.5) with initial condition $y$. It is well-known that the strong approximation error of the Euler-Maruyama scheme $\mathbb{E}\left[\left|X_{1}^{x}-X^{x}\right|^{\kappa}\right]^{1 / \kappa} \leq C h_{l}^{1 / 2}$ for $\kappa>0$ (see for example $[25,27])$, where $X^{x}$ is also correlated to $X_{1}^{x}$, in the sense that the latter arises from a coarsening like (3.5) except with an integration of the stochastic forcing $\xi(t)$ over the interval $h_{l}$. Let us generalize this slightly and assume some approximation method for which

$$
\mathbb{E}\left[\left|X_{1}^{x}-X^{x}\right|^{\kappa}\right]^{1 / \kappa} \leq C h_{l}^{\beta / 2}
$$

for some $\beta>0$ and for any $x \in \mathbb{R}^{d}$ for some $C>0$. As mentioned above, the equation holds for $\beta=1$ for the Euler-Maruyama scheme under Assumption 2.1.

Proposition D.1. Assume Assumption 2.1 and (D.1) for $\beta>0$. Now let $y \in \mathbb{R}^{d}$. Then there exists a $C^{\prime}>0$ such that

$$
\mathbb{E}\left[\left|X_{1}^{x}-X_{2}^{y}\right|^{\kappa}\right]^{1 / \kappa} \leq C^{\prime}\left(|x-y|+h_{l}^{\beta / 2}\right) .
$$

Proof. By the triangular inequality, it is sufficient to show

$$
\begin{aligned}
& \mathbb{E}\left[\left|X_{1}^{x}-X^{x}\right|^{\kappa}\right]^{1 / \kappa} \leq C h_{l}^{\beta / 2} \\
& \mathbb{E}\left[\left|X^{x}-X^{y}\right|^{\kappa}\right]^{1 / \kappa} \leq C^{\prime}|x-y|,
\end{aligned}
$$

The first inequality holds by assumption. Now note that Assumption 4.1 follows from Corollary V.11.7 of [31] together with Grönwall's inequality, and the second estimate is immediate.

Note that this provides Assumption 4.2(ii). For Euler the rate $\beta=1$ is well-known and may be found for example in [25, 27]. Assume $M_{n, 1}^{l}$ and $M_{n, 2}^{l}$ are transition kernels corresponding to Euler-Maruyama scheme with grid sizes $h_{l}$ and $h_{l-1}$ respectively. Then, under the uniformly elliptic condition Assumption 2.1(i), by equation (2.4) of [11],

$$
||\left|M_{n, 1}^{l}-M_{n, 2}^{l}\right||| \leq C h_{l}^{\alpha}
$$

for $\alpha=1$. This shows that the second term in Assumption 4.2(i) provides $\alpha=1$. As for the first term of Assumption 4.2(i), preservation of the weak errror, the reader is referred to $[27,16]$ where appropriate assumptions are detailed. Now an inequality for predictors can be proven.

Lemma D.1. Assume Assumptions 2.1, 2.2 and (D.2) for $\alpha>0$. For $l, n \in \mathbb{N}$, there exists $C>0$ such that

$$
\left\|\eta_{n, 1}^{l}-\eta_{n, 2}^{l}\right\|_{\mathrm{tv}} \leq C h_{l}^{\alpha}
$$


Proof. Let

$\left(H_{n, 1}^{l} \varphi\right)(x)=\int M_{n, 1}^{l}\left(x, d x^{*}\right) G_{n-1}(x) \varphi\left(x^{*}\right),\left(H_{n, 2}^{l} \varphi\right)(x)=\int M_{n, 2}^{l}\left(x, d x^{*}\right) G_{n-1}(x) \varphi\left(x^{*}\right)$.

Then

$$
\eta_{n, 1}^{l} \varphi=\frac{\eta_{n-1,1}^{l} H_{n, 1}^{l} \varphi}{\eta_{n-1,1}^{l} H_{n, 1}^{l} 1}, \eta_{n, 2}^{l} \varphi=\frac{\eta_{n-1,2}^{l} H_{n, 2}^{l} \varphi}{\eta_{n-1,2}^{l} H_{n, 2}^{l} 1} .
$$

By definition, $\eta_{0,1}^{l}=\eta_{0,2}^{l}$. Suppose that the claim holds for $0,1, \ldots, n-1$. Then

$$
\begin{aligned}
\left|\eta_{n, 1}^{l} \varphi-\eta_{n, 2}^{l} \varphi\right|= & \left|\frac{\eta_{n-1,1}^{l} H_{n, 1}^{l} \varphi}{\eta_{n-1,1}^{l} H_{n, 1}^{l} 1}-\frac{\eta_{n-1,2}^{l} H_{n, 2}^{l} \varphi}{\eta_{n-1,2}^{l} H_{n, 2}^{l} 1}\right| \\
\leq & \frac{1}{\eta_{n-1,1}^{l} H_{n, 1}^{l} 1}\left|\eta_{n-1,1}^{l} H_{n, 1}^{l} \varphi-\eta_{n-1,2}^{l} H_{n, 2}^{l} \varphi\right| \\
& +\frac{\eta_{n-1,2}^{l} H_{n, 2}^{l} \varphi}{\eta_{n-1,1}^{l} H_{n, 1}^{l} 1 \times \eta_{n-1,2}^{l} H_{n, 2}^{l} 1}\left|\eta_{n-1,1}^{l} H_{n, 1}^{l} 1-\eta_{n-1,2}^{l} H_{n, 2}^{l} 1\right| .
\end{aligned}
$$

By Assumption 2.2(i), $c^{-1} \leq \eta_{n-1,1}^{l} H_{n, 1}^{l} 1, \eta_{n-1,2}^{l} H_{n, 2}^{l} 1 \leq c$. Thus it is sufficient to show

$$
\left|\eta_{n-1,1}^{l} H_{n, 1}^{l} \varphi-\eta_{n-1,2}^{l} H_{n, 2}^{l} \varphi\right| \leq C\|\varphi\| h_{l}^{\alpha} .
$$

However, the left-hand side of the above is dominated by

$$
\begin{aligned}
& \left|\eta_{n-1,1}^{l} H_{n, 1}^{l} \varphi-\eta_{n-1,2}^{l} H_{n, 1}^{l} \varphi\right|+\left|\eta_{n-1,2}^{l} H_{n, 1}^{l} \varphi-\eta_{n-1,2}^{l} H_{n, 2}^{l} \varphi\right| \\
& \leq\left(\left\|\eta_{n-1,1}^{l}-\eta_{n-1,2}^{l}\right\|_{t v}+|| M_{n, 1}^{l}-M_{n, 2}^{l}|| \mid\right) \sup _{x, y}|G(y, x)|\|\varphi\| \leq C\|\varphi\| h_{l}^{\alpha} .
\end{aligned}
$$

where the second inequality follows from the induction assumption, and Assumption 2.2(i) and (D.2). Thus the claim follows by induction. $\square$

Let $I_{n, 1}^{l}(k):=I_{n, 1}^{l, k}$ and $I_{n, 2}^{l}(k):=I_{n, 2}^{l, k}$. For $n \geq 2$, let $S_{n}^{l}$ be the indices that choose the same ancestor in each resampling step, that is,

$$
\begin{gathered}
S_{n}^{l}=\left\{k \in\left\{1, \ldots, N_{l}\right\} ; I_{n, 1}^{l}(k)=I_{n, 2}^{l}(k), I_{n-1,1}^{l} \circ I_{n, 1}^{l}(k)=I_{n-1,2}^{l} \circ I_{n, 2}^{l}(k), \cdots,\right. \\
\left.I_{1,1}^{l} \circ I_{2,1}^{l} \circ \cdots \circ I_{n, 1}^{l}(k)=I_{1,2}^{l} \circ \cdots \circ I_{2,2}^{l} \circ I_{n, 2}^{l}(k)\right\} .
\end{gathered}
$$

For $n=1$, set $S_{1}^{l}=\left\{1, \ldots, N_{l}\right\}$. Let

$\mathcal{F}_{n}^{l}=\sigma\left(\left\{U_{p, 1}^{l, k}, U_{p, 2}^{l, k}, \widehat{U}_{p, 1}^{l, k}, \widehat{U}_{p, 2}^{l, k}, I_{p, 1}^{l}, I_{p, 2}^{l} ; p<n, k \leq N_{l}\right\} \cup\left\{U_{n, 1}^{l, k}, U_{n, 2}^{l, k}, k \leq N_{l}\right\}\right)$,

$\widehat{\mathcal{F}}_{n}^{l}=\sigma\left(\left\{U_{p, 1}^{l, k}, U_{p, 2}^{l, k}, \widehat{U}_{p, 1}^{l, k}, \widehat{U}_{p, 2}^{l, k}, I_{p, 1}^{l}, I_{p, 2}^{l} ; p<n, k \leq N_{l}\right\} \cup\left\{U_{n, 1}^{l, k}, U_{n, 2}^{l, k}, \widehat{U}_{n, 1}^{l, k}, \widehat{U}_{n, 2}^{l, k}, k \leq N_{l}\right\}\right)$.

Lemma D.2. Assume Assumptions 2.1, 2.2 and (D.1) for $\beta>0$. For $\kappa>0$ and $n \in \mathbb{N}$, there exists $C>0$ such that

$$
\mathbb{E}\left[\frac{1}{N_{l}} \sum_{k \in S_{n-1}^{l}}\left|U_{n, 1}^{l, k}-U_{n, 2}^{l, k}\right|^{\kappa}\right]^{1 / \kappa} \leq C h_{l}^{\beta / 2} .
$$


Proof. By Proposition D.1,

$$
\begin{aligned}
\mathbb{E}\left[\frac{1}{N_{l}} \sum_{k \in S_{n-1}^{l}}\left|U_{n, 1}^{l, k}-U_{n, 2}^{l, k}\right|^{\kappa}\right]^{1 / \kappa} & =\mathbb{E}\left[\frac{1}{N_{l}} \sum_{k \in S_{n-1}^{l}} \mathbb{E}\left[\left|U_{n, 1}^{l, k}-U_{n, 2}^{l, k}\right|^{\kappa} \mid \widehat{\mathcal{F}}_{n-1}^{l}\right]\right]^{1 / \kappa} \\
& \leq C \mathbb{E}\left[\frac{1}{N_{l}} \sum_{k \in S_{n-1}^{l}}\left\{\left|\widehat{U}_{n-1,1}^{l, k}-\widehat{U}_{n-1,2}^{l, k}\right|+h_{l}^{\beta / 2}\right\}^{\kappa}\right]^{1 / \kappa}
\end{aligned}
$$

Since $(a+b)^{\kappa} \leq C\left(a^{\kappa}+b^{\kappa}\right)(a, b \geq 0)$, we have

$$
\begin{aligned}
\mathbb{E}\left[\frac{1}{N_{l}} \sum_{k \in S_{n-1}^{l}}\left|U_{n, 1}^{l, k}-U_{n, 2}^{l, k}\right|^{\kappa}\right]^{1 / \kappa} & \leq C \mathbb{E}\left[\frac{1}{N_{l}} \sum_{k \in S_{n-1}^{l}}\left|\widehat{U}_{n-1,1}^{l, k}-\widehat{U}_{n-1,2}^{l, k}\right|^{\kappa}\right]^{1 / \kappa}+C h_{l}^{\beta / 2} \\
& =C \mathbb{E}\left[\frac{1}{N_{l}} \sum_{k \in S_{n-1}^{l}}\left|U_{n-1,1}^{l, I_{n-1}^{l, k}}-U_{n-1,2}^{l, I_{n-1,2}^{l, k}}\right|^{\kappa}\right]^{1 / \kappa}+C h_{l}^{\beta / 2}
\end{aligned}
$$

Note that $I_{n-1,1}^{l}=I_{n-1,2}^{l}$ for $k \in S_{n-1}^{l}$. The conditional distribution of $\left(U_{n-1,1}^{l, I_{n-1,1}^{l, k}}, U_{n-1,2}^{l, I_{n-1,2}^{l, k}}\right)(k \in$ $\left.S_{n-1}^{l}\right)$ given $S_{n-1}^{l}$ and $\mathcal{F}_{n-1}^{l}$ is

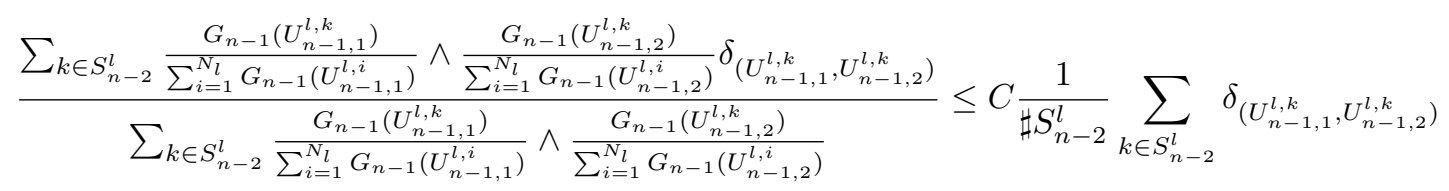

The expected value of $\sharp S_{n-1}^{l}$ given $\mathcal{F}_{n-1}^{l}$ is

$$
\mathbb{E}\left[\frac{\sharp S_{n-1}^{l}}{N_{l}} \mid \mathcal{F}_{n-1}^{l}\right]=\sum_{k \in S_{n-2}^{l}} \frac{G_{n-1}\left(U_{n-1,1}^{l, k}\right)}{\sum_{i=1}^{N_{l}} G_{n-1}\left(U_{n-1,1}^{l, i}\right)} \wedge \frac{G_{n-1}\left(U_{n-1,2}^{l, k}\right)}{\sum_{i=1}^{N_{l}} G_{n-1}\left(U_{n-1,2}^{l, i}\right)} \leq C \frac{\sharp S_{n-2}^{l}}{N_{l}} .
$$


Therefore

$$
\begin{aligned}
& \mathbb{E}\left[\frac{1}{N_{l}} \sum_{k \in S_{n-1}^{l}}\left|U_{n-1,1}^{l, I_{n-1,1}^{l, k}}-U_{n-1,2}^{l, I_{n-1,2}^{l, k}}\right|^{\kappa}\right] \\
& =\mathbb{E}\left[\frac { 1 } { N _ { l } } \sum _ { k \in S _ { n - 1 } ^ { l } } \mathbb { E } \left[\mid U_{n-1,1}^{\left.\left.l, I_{n-1,1}^{l, k}-\left.U_{n-1,2}^{l, I_{n-1,2}^{l, k}}\right|^{\kappa} \mid S_{n-1}^{l}, \mathcal{F}_{n-1}^{l}\right]\right]}\right.\right. \\
& =\mathbb{E}\left[\frac{\sharp S_{n-1}^{l}}{N_{l}}\left\{\frac{\sum_{k \in S_{n-2}^{l}}\left|U_{n-1,1}^{l, k}-U_{n-1,2}^{l, k}\right|^{\kappa} \frac{G_{n-1}\left(U_{n-1,1}^{l, k}\right)}{\sum_{i=1}^{N_{l}} G_{n-1}\left(U_{n-1,1}^{l, i}\right)} \wedge \frac{G_{n-1}\left(U_{n-1,2}^{l, k}\right)}{\sum_{i=1}^{N} G_{n-1}\left(U_{n-1,2}^{l, i}\right)}}{\sum_{k \in S_{n-2}^{l}} \frac{G_{n-1}\left(U_{n-1,1}^{l, k}\right)}{\sum_{i=1}^{N_{l}} G_{n-1}\left(U_{n-1,1}^{l, i}\right)} \wedge \frac{G_{n-1}\left(U_{n-1,2}^{l, k}\right)}{\sum_{i=1}^{N_{l}} G_{n-1}\left(U_{n-1,2}^{l, i}\right)}}\right\}\right]
\end{aligned}
$$

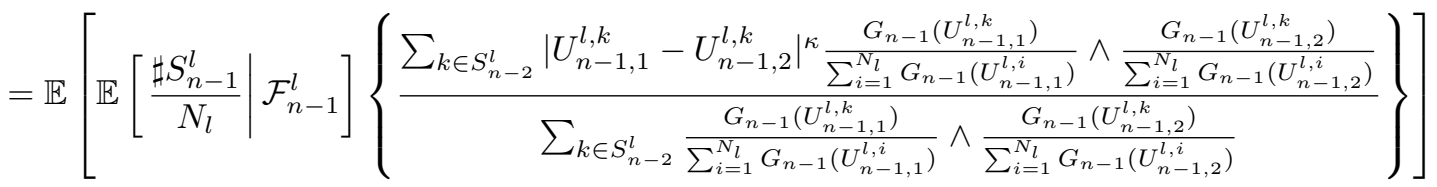

$$
\begin{aligned}
& \leq C \mathbb{E}\left[\frac{1}{N_{l}} \sum_{k \in S_{n-2}^{l}}\left|U_{n-1,1}^{l, k}-U_{n-1,2}^{l, k}\right|^{\kappa}\right] \text {. }
\end{aligned}
$$

Thus the claim comes from induction. $\square$

Lemma D.3. Under Assumptions 2.1, 2.2 and (D.1) for $\beta>0$, there exists $C>0$ such that for $n \in \mathbb{N}$,

$$
1-\mathbb{E}\left[\frac{\sharp S_{n}^{l}}{N_{l}}\right] \leq C h_{l}^{\beta / 2} .
$$

Proof. Note that

$$
\begin{aligned}
1-\sum_{k=1}^{N_{l}} \frac{G_{n}\left(U_{n, 1}^{l, k}\right)}{\sum_{i=1}^{N_{l}} G_{n}\left(U_{n, 1}^{l, i}\right)} \wedge \frac{G_{n}\left(U_{n, 2}^{l, k}\right)}{\sum_{i=1}^{N_{l}} G_{n}\left(U_{n, 2}^{l, i}\right)} & =\frac{1}{2} \sum_{k=1}^{N_{l}}\left|\frac{G_{n}\left(U_{n, 1}^{l, k}\right)}{\sum_{i=1}^{N_{l}} G_{n}\left(U_{n, 1}^{l, i}\right)}-\frac{G_{n}\left(U_{n, 2}^{l, k}\right)}{\sum_{i=1}^{N_{l}} G_{n}\left(U_{n, 2}^{l, i}\right)}\right| \\
& \leq \frac{1}{2} \sum_{k \in S_{n-1}^{l}}\left|\frac{G_{n}\left(U_{n, 1}^{l, k}\right)}{\sum_{i=1}^{N_{l}} G_{n}\left(U_{n, 1}^{l, i}\right)}-\frac{G_{n}\left(U_{n, 2}^{l, k}\right)}{\sum_{i=1}^{N_{l}} G_{n}\left(U_{n, 2}^{l, i}\right)}\right| \\
& +\frac{1}{2} \sum_{k \notin S_{n-1}^{l}}\left|\frac{G_{n}\left(U_{n, 1}^{l, k}\right)}{\sum_{i=1}^{N_{l}} G_{n}\left(U_{n, 1}^{l, i}\right)}-\frac{G_{n}\left(U_{n, 2}^{l, k}\right)}{\sum_{i=1}^{N_{l}} G_{n}\left(U_{n, 2}^{l, i}\right)}\right| \\
\leq & \frac{1}{N_{l}} \sum_{k \in S_{n-1}^{l}}\left|U_{n, 1}^{l, k}-U_{n, 2}^{l, k}\right|+C\left(1-\frac{\sharp S_{n-1}^{l}}{N_{l}}\right) .
\end{aligned}
$$


Thus we have

$$
\begin{aligned}
\left(1-\mathbb{E}\left[\frac{\sharp S_{n}^{l}}{N_{l}} \mid \mathcal{F}_{n}^{l}\right]\right)= & \left\{1-\sum_{k=1}^{N_{l}} \frac{G_{n}\left(U_{n, 1}^{l, k}\right)}{\sum_{i=1}^{N_{l}} G_{n}\left(U_{n, 1}^{l, i}\right)} \wedge \frac{G_{n}\left(U_{n, 2}^{l, k}\right)}{\sum_{i=1}^{N_{l}} G_{n}\left(U_{n, 2}^{l, i}\right)}\right\} \\
& +\sum_{k \notin S_{n-1}^{l}} \frac{G_{n}\left(U_{n, 1}^{l, k}\right)}{\sum_{i=1}^{N_{l}} G_{n}\left(U_{n, 1}^{l, i}\right)} \wedge \frac{G_{n}\left(U_{n, 2}^{l, k}\right)}{\sum_{i=1}^{N_{l}} G_{n}\left(U_{n, 2}^{l, i}\right)} \\
\leq & C \frac{1}{N_{l}} \sum_{k \in S_{n-1}^{l}}\left|U_{n, 1}^{l, k}-U_{n, 2}^{l, k}\right|+C\left(1-\frac{\sharp S_{n-1}^{l}}{N_{l}}\right) .
\end{aligned}
$$

The claim follows by induction. $\square$

Theorem D.1. Assume Assumptions 2.1, 2.2 and (D.1) for $\beta>0$. For $\kappa>1$ and $n \in \mathbb{N}$, there exists $C>0$ such that

$$
\mathbb{E}\left[\left(\left|U_{n, 1}^{l, 1}-U_{n, 2}^{l, 1}\right| \wedge 1\right)^{\kappa}\right]^{1 / \kappa} \leq C h_{l}^{\beta / 2 \kappa}
$$

Proof. By Lemmas D.2 and D.3,

$$
\begin{aligned}
\mathbb{E}\left[\left(\left|U_{n, 1}^{l, 1}-U_{n, 2}^{l, 1}\right| \wedge 1\right)^{\kappa}\right] & =\mathbb{E}\left[\frac{1}{N_{l}} \sum_{k=1}^{N_{l}}\left(\left|U_{n, 1}^{l, k}-U_{n, 2}^{l, k}\right| \wedge 1\right)^{\kappa}\right] \\
& =\mathbb{E}\left[\frac{1}{N_{l}} \sum_{k \in S_{n-1}^{l}}\left(\left|U_{n, 1}^{l, k}-U_{n, 2}^{l, k}\right| \wedge 1\right)^{\kappa}\right]+\mathbb{E}\left[\frac{1}{N_{l}} \sum_{k \notin S_{n-1}^{l}}\left(\left|U_{n, 1}^{l, k}-U_{n, 2}^{l, k}\right| \wedge 1\right)^{\kappa}\right] \\
& \leq C h_{l}^{\kappa \beta / 2}+C h_{l}^{\beta / 2} \leq 2 C h_{l}^{\beta / 2} .
\end{aligned}
$$

Thus the claim follows. $\square$

Let $\alpha, \beta, \gamma>0$ be as in Assumption 4.2, and let $L=L(\epsilon) \propto-\log (\epsilon) /(\log (2) \alpha)$, $K=K(\epsilon)=\sum_{l=0}^{L} \sqrt{V_{l} C_{l}}, C_{l} \propto h_{l}^{-\gamma}, V_{l} \propto h_{l}^{\beta / 2}, N_{l} \propto \epsilon^{-2} K(\epsilon) 2^{-(\beta+2 \gamma) l / 4}$ and $h_{l}=2^{-l}$ as in Section 3.1.

Corollary D.1. Assume Assumptions 2.1, 2.2 and 4.2. If $\gamma / \alpha \leq 2$, then the bound of Theorem 4.2 is dominated by

$$
\sum_{l=0}^{L} \frac{C(m, \varphi)}{N_{l}} h_{l}^{\beta / 2}
$$

where $C(m, \varphi)=\max _{0 \leq l \leq L} C_{l}(m, \varphi)$.

Proof. First note that Theorem D.1 provides a bound of $C_{l}(m, \varphi) h_{l}^{\beta / 2}$ on the first term of $B_{l}(n)$ defined in (C.1), and other terms are bounded by $C_{l}(m, \varphi) h_{l}^{2 \alpha}$. Recall that $2 \alpha \geq \beta$, as they are defined here.

Now, one must show that $\sum_{l=0}^{L} \frac{\sqrt{B_{l}}}{N_{l}} \sum_{q=0 \neq l}^{L} \frac{\sqrt{B_{q}}}{N_{q}}$ is higher order in comparison to $\sum_{l=0}^{L} \frac{B_{l}}{N_{l}}=\mathcal{O}\left(\varepsilon^{2}\right)$. Choosing $L(\varepsilon)$ and $K(\varepsilon)$ as described in Section 3.1 and the proof of Theorem 4.1, one has

$$
\sum_{l=0}^{L} \frac{\sqrt{B_{l}}}{N_{l}} \sum_{q=0 \neq l}^{L} \frac{\sqrt{B_{q}}}{N_{q}} \lesssim \varepsilon^{4} K(\varepsilon)^{-2} \sum_{l=0}^{L} \sqrt{C_{l}} \sum_{q=0 \neq l}^{L} \sqrt{C_{q}} .
$$


Notice each of the two summations is $\mathcal{O}\left(C_{L}\right)=\mathcal{O}\left(\varepsilon^{-\gamma / 2 \alpha}\right)$, and $K(\varepsilon)^{-1}=O(1)$. Therefore,

$$
\sum_{l=0}^{L} \frac{\sqrt{B_{l}}}{N_{l}} \sum_{q=0 \neq l}^{L} \frac{\sqrt{B_{q}}}{N_{q}} \lesssim \varepsilon^{2} \varepsilon^{2-\gamma / \alpha}
$$

and under the assumption that $\gamma / \alpha \leq 2$ the proof is concluded.

\section{REFERENCES}

[1] Bengtsson, T., Bickel, P. \& Li, B. (2008), Curse-of-dimensionality revisited: Collapse of the particle filter in very large scale systems, Probability and statistics: Essays in honor of David A. Freedman, 316-334. Institute of Mathematical Statistics.

[2] Bengtsson, T., Bickel, P. \& Li, B. (2008). Sharp failure rates for the bootstrap particle filter in high dimensions. Pushing the limits of contemporary statistics: Contributions in honor of Jayanta K. Ghosh, 318-329. Institute of Mathematical Statistics.

[3] Beskos, A., Jasra, A., Law, K., Tempone, R., \& Zhou, Y. (2017). Multilevel Sequential Monte Carlo Samplers. Stoch. Proc. Appl., 127, 1417-1440.

[4] Briggs, W. L. \& McCormick, S. F. (2000). A Multigrid Tutorial. SIAM: Philadelphia.

[5] Cappé, O., Ryden, T, \& Moulines, É. (2005). Inference in Hidden Markov Models. Springer: New York.

[6] Chopin, N., \& Singh, S.S. (2015) On particle Gibbs sampling. Bernoulli, 21, 1855 ?1883.

[7] Del Moral, P. (2004). Feynman-Kac Formulae: Genealogical and Interacting Particle Systems with Applications. Springer: New York.

[8] Del Moral, P. (2013). Mean Field Simulation for Monte Carlo Integration Chapman \& Hall: London.

[9] Del Moral, P., \& Guionnet, A. (2001). On the stability of interacting processes with applications to filtering and genetic algorithms. Ann. Inst. Henri Poincaire, 37, 155-194.

[10] Del Moral, P., Doucet, A. \& Jasra, A. (2012) On Adaptive Resampling Procedures for SMC Methods, Bernoulli, 18, 252-272.

[11] Del Moral, P., Jacod, J. \& Protter, P. (2001) The Monte-Carlo method for filtering with discrete-time observations. Probab. Theory Rel. Fields, 120, 346-368.

[12] Doucet, A. \& Johansen, A. (2011). A tutorial on particle filtering and smoothing: Fifteen years later. In Handbook of Nonlinear Filtering (eds. D. Crisan \& B. Rozovsky), Oxford University Press: Oxford.

[13] Fearnhead, P., Papaspiliopoulos, O. \& Roberts, G. O. (2008). Particle filters for partially observed diffusions. J. R. Stat. Soc. Ser. B 70, 755-777.

[14] Giles, M. B. (2008). Multi-level Monte Carlo path simulation. Op. Res., 56, 607-617.

[15] Giles, M. B. (2015) Multilevel Monte Carlo methods. Acta Numerica 24, 259-328.

[16] Graham, C. \& Talay, D. (2013). Stochastic Simulation and Monte Carlo methods. Springer: Heidelberg.

[17] Gregory, A., \& Cotter, C. (2016). A seamless multilevel ensemble transform particle filter. arXiv preprint arXiv:1611.00266.

[18] Gregory, A., Cotter, C., \& Reich, S. (2016). Multilevel Ensemble Transform Particle Filtering. SIAM J. Sci. Comp. 38, A1317-A1338

[19] Heinrich, S. (2001). Multilevel monte carlo methods. In Large-Scale Scientific Computing, (eds. S. Margenov, J. Wasniewski \& P. Yalamov), Springer: Berlin.

[20] Hoang, V. H., Schwab, C, \& Stuart, A.M. (2013). Complexity analysis of accelerated MCMC methods for Bayesian inversion. Inverse Problems 29:8, 085010.

[21] Hoel, H., Law, K.J.H. \& Tempone, R. (2016) Multilevel ensemble Kalman filtering. SIAM Journal on Numerical Analysis, 54.3, 1813-1839.

[22] Jasra, A. \& Doucet, A. (2009). Sequential Monte Carlo for diffusion processes. Proc. Roy Soc. A, 465, 3709-3727.

[23] Jasra, A., Kamatani, K., Law, K. J., \& Zhou, Y. (2015). Multilevel particle filters. arXiv preprint arXiv:1510.04977.

[24] Jasra, A., Kamatani, K., Osei, P. P., \& Zhou, Y. (2017). Multilevel particle filters: Normalizing constant estimation. Statist. Comp. (to appear).

[25] Jourdain, B. \& Kohatsu-Higa, A. (2011) A review of recent results on approximation of solutions of stochastic differential equations. Stoch. Anal. Finan. Appl.. 65, 121-144. 
[26] Ketelsen, C., Scheichl, R. \& Teckentrup, A. L. (2015). A hierarchical multilevel Markov chain Monte Carlo algorithm with applications to uncertainty quantification in subsurface flow. SIAM/ASA J. Uncer. Quant., 3, 1075-1108.

[27] Kloeden, P. E. \& Platen. E (1992). Numerical Solution of Stochastic Differential Equations. Springer: Berlin.

[28] Law, K., Stuart, A. And Zygalakis, K. (2015). Data Assimilation: A Mathematical Introduction. Springer Texts in Applied Mathematics, vol. 62.

[29] Oksendal, B. (1995) Stochastic Differential Equations: An Introduction with Applications. Springer: New York.

[30] Sen, D., Thiery, A. \& Jasra, A. (2017). On coupling particle filter trajectories. Statist. Comp. (to appear).

[31] Rogers, L. C. G. \& Williams, D. (2000) Diffusions, Markov Processes and Martingales. Vol. 2. CUP: Cambridge. 